\title{
Light dark matter candidates in intense laser pulses II: the relevance of the spin degrees of freedom
}

\section{S. Villalba-Chávez and C. Müller}

Institut für Theoretische Physik I, Heinrich-Heine-Universität Düsseldorf

Universitätsstr. 1, 40225 Düsseldorf, Germany

E-mail: selym@tp1.uni-duesseldorf.de,

c.mueller@tp1.uni-duesseldorf.de

ABstRAct: Optical searches assisted by the field of a laser pulse might allow for exploring a variety of not yet detected dark matter candidates such as hidden-photons and scalar minicharged particles. These hypothetical degrees of freedom may be understood as a natural consequence of extensions of the Standard Model incorporating a hidden $\mathrm{U}(1)$-gauge sector. In this paper, we study the effects induced by both candidates on the propagation of a probe electromagnetic wave in the vacuum polarized by a long laser pulse of moderate intensity, this way complementing our previous study [JHEP 06 (2015) 177]. We describe how the absence of a spin in the scalar charged carriers modifies the photonparaphoton oscillations as compared with a fermionic minicharge model. In particular, we find that the regime close to their lowest threshold mass might provide the most stringent upper limit for minicharged scalars. The pure-laser based experiment investigated here could allow for excluding a sector in the parameter space of the particles which has not been experimentally ruled out by setups driven by dipole magnets. We explain how the sign of the ellipticity and rotation of the polarization plane acquired by a probe photon - in combination with their dependencies on the pulse parameters - can be exploited to elucidate the quantum statistics of the charge carriers.

Keywords: Beyond Standard Model, Electromagnetic Processes and Properties

ARXIV EPRINT: 1510.00222 


\section{Contents}

1 Introduction 1

2 Theoretical aspects 3

2.1 Photon Green's function and vacuum polarization 3

2.2 Optical observables: including the paraphoton interplay 5

2.3 Absorption coefficients and refractive indices at $\xi_{\epsilon}<1 \quad 8$

3 Experimental prospects $\quad 10$

$\begin{array}{lll}3.1 & \text { Estimating the exclusion limits } & 10\end{array}$

3.2 Characteristic of the signals in the scalar MCPs model 14

4 Conclusions and outlook $\quad 19$

\section{Introduction}

Identifying the dark matter in the Universe and consistently incorporating it into the Standard Model (SM) constitute challenging problems in today's particle physics. Cosmological as well as astrophysical observations provide substantial evidence that only a small fraction $4,5 \%$ of matter is made out of the elementary building blocks of the SM but there is not yet a clear idea about the origin and nature of the dark matter [1-4]. This fact evidences why the SM is currently accepted as an effective theory which must be embedded into a more general framework at higher energy scales. Such an enlarged theory is expected to offer us a comprehensive theoretical understanding about a variety of central problems including the charge quantization, which presently lacks an experimentally verifiable explanation. While some extensions of the SM provide mechanisms for enforcing charge quantization, other scenarios including carriers of small unquantized charge are not discarded. Indeed, effective theories containing an extra U(1) gauge field [5-8] kinematically mixed with the electromagnetic sector [9-12], introduce this sort of Mini-Charged Particles (MCPs) [1315] in a natural way. The fact that at low energies these carriers are not observed might be considered as an evidence indicating that the sector to which they belong interacts only very weakly with the well established SM branch. It is, in addition, reasonable to assume that a hypothetical existence of MCPs induces nonlinear interactions in the electromagnetic field provided they are very light sub-eV particles minimally coupled to the "visible" $\mathrm{U}(1)$ sector $[16,17]$. Slight discrepancies are expected then as compared with the inherent phenomenology of Quantum Electrodynamics (QED). Indeed, motivated by this possibility, various experimental collaborations have imposed constraints and ruled out sectors in the parameter space of these hypothetical degrees freedom. 
The phenomena of interest which have been exploited in this research area so far are summarized in several reviews [18-21]. These searches fall into two categories depending upon the scenario under consideration. On the one side, there are searches relying on astro-cosmological observations. They provide the most stringent constraints at present. Indeed, arguments related to energy loss which is not observed in Horizontal Branch stars, limit the relative charge in MCPs to $\epsilon \lesssim 10^{-14}$ for masses below a few $\mathrm{keV}$ [22]. However, further investigations in this direction have provided arguments indicating the extent to which this bound is sensitive to the inclusion of macroscopic and microscopic parameters of the star, as well as to certain processes that might attenuate it significantly and, simultaneously, elude it from our perception [23-26]. The described vulnerability in the astro-cosmological constraints is a strong motivation for considering, on the other side, well-controlled laboratory-based searches as a complementary approach. Generally, these have been conducted through high precision experiments looking for the birefringence and dichroism of the vacuum ${ }^{1}$ [30-34], modifications in Coulomb's law [35, 36] or through the regeneration of photons from a hidden photon field in "Light Shining Through a Wall" setups [37-42]. For details, variants and prospects of this kind of experiment we refer also to refs. [43-48]. Most of these experiments require the presence of a static external magnetic field to induce vacuum polarization mediated by virtual pairs of MCPs. As a general rule, the relevant observables depend on the field strength as well as its spatial extend and, usually, such dependencies allow for finding more stringent bounds as both parameters increase. However, today our technical capability in laboratories are quite limited, allowing us to achieve constant magnetic fields no higher than $\sim 10^{5} \mathrm{G}$ along effective distances of the order of $\sim 1 \mathrm{~km}$.

Focused laser pulses of few micrometer extension can produce much stronger magnetic fields but they are inhomogeneously distributed [49]. For instance, the highest peak intensity achieved so far $2 \times 10^{22} \mathrm{~W} / \mathrm{cm}^{2}$ [50] corresponds to a magnetic field strength of $9 \times 10^{9} \mathrm{G}$. Besides, peak magnetic fields exceeding $\sim 10^{11} \mathrm{G}$ are likely to be reached by the ongoing ELI and XCELS projects [51, 52], in which intensities greater than $10^{25} \mathrm{~W} / \mathrm{cm}^{2}$ are envisaged. In view of these perspectives, high-intensity laser pulses are potential tools with which nonlinear phenomena in strong field QED [53-56] can be observed for the first time. Obviously, this would also provide an opportunity for detecting the birefringence of the vacuum [57]. ${ }^{2}$ Indeed, motivated by this idea, the HIBEF consortium has proposed a laser-based experiment which combines a Petawatt optical laser with a x-ray free electron laser [60]. Similarly to setups driven by static magnetic fields, polarimetric experiments assisted by an external laser-wave might also constitute a sensitive probe for searching weakly interacting particles. Although studies of this nature have been put forward for the case of axion-like particles [17,61-65], the estimate of the exclusion limits for MCPs and hidden photon fields from laser-based polarimetric searches is much less developed.

A first study on MCPs has been given by the authors in ref. [66]. Later, in part I of this series [67], a further step was performed by investigating the optical effects resulting

\footnotetext{
${ }^{1}$ Recent theoretical studies on the birefringence and dichroism of the vacuum in an external magnetic field can be found in [27-29].

${ }^{2}$ See $[58,59,66,67]$ for new insights on the vacuum refractive indices in plane-wave fields.
} 
from an extended model containing fermionic MCPs and a hidden photon field. There we revealed that, at moderate intensities $\lesssim 10^{16} \mathrm{~W} / \mathrm{cm}^{2}$ as provided by the nanosecond frontends of the PHELIX laser [71] and LULI system [72], high-precision polarimetric measurements could improve the existing laboratory upper bounds for the coupling constant of MCPs by an order of magnitude for masses of the order of $m_{\epsilon} \sim \mathrm{eV}$. However, charge carriers with unquantized electric charges might be realized in nature not only as fermions but also as scalar particles [73]. Hence, a complete study of this subject requires in addition the insights coming from the polarization tensor [68-70] that results from the Green's function of scalar MCPs and in which the field of the wave is incorporated in full glory. For a monochromatic plane-wave background, corresponding expressions in a pure QED context have been obtained previously $[68,74]$. In this paper, we study the effects resulting from scalar minicharges and paraphotons in a plausible polarimetric setup assisted by a long laser pulse of moderate intensity. We show how the absence of spin in the scalar charge carriers modifies the photon-paraphoton oscillations as compared with a fermionic minicharges model. In particular, we explain how the sign of the ellipticity and rotation of the polarization plane acquired by a probe photon beam - in combination with their dependencies on the pulse parameters - can be exploited to elucidate the quantum statistics of MCPs.

\section{Theoretical aspects}

\subsection{Photon Green's function and vacuum polarization}

It is a long-standing prediction of QED that the optical properties of its vacuum are modified in the presence of an external electromagnetic field due to the nontrivial interplay between photons and the fluctuations of virtual electron-positron pairs polarized by an external field. Indeed, compelling theoretical studies provide evidences for self-coupling of photons, rendering QED a nonlinear theory which allows for birefringence and absorption of photons traveling through the polarized region of the vacuum. However, the source of fluctuations inducing nonlinear self-interactions of the electromagnetic field is not restricted to virtual electrons and positrons. Although at the energy scale of QED, the structure of the quantum vacuum is mainly determined by these virtual entities, actually any quantum degree of freedom that couples to photons modifies the structure of the effective vertices which result from the generating functional of the one-particle irreducible Feynman graphs. The lowest one, i.e., the one containing two amputated legs: ${ }^{3}$

$$
\mathscr{D}_{\mu \nu}^{-1}\left(k, k^{\prime}\right)=-\frac{1}{4 \pi}\left(k^{2} g_{\mu \nu}-k_{\mu} k_{\nu}\right) \delta_{k, k^{\prime}}+\frac{1}{4 \pi} \Pi_{\mu \nu}\left(k, k^{\prime}\right),
$$

defines the vacuum polarization tensor $\Pi_{\mu \nu}\left(k, k^{\prime}\right)$ through the Green's function of MCPs as well as the bare and dressed vertices, as it occurs in a pure QED context. Here $g_{\mu \nu}=$ $\operatorname{diag}(+1,-1,-1,-1)$ denotes the flat metric tensor, whereas the shorthand notation $\delta_{k, k^{\prime}}=$ $(2 \pi)^{4} \delta^{4}\left(k-k^{\prime}\right)$ has been introduced.

\footnotetext{
${ }^{3}$ From now on "natural" and Gaussian units with $c=\hbar=4 \pi \epsilon_{0}=1$ are used.
} 
In the one-loop approximation, and in the field of a circularly polarized monochromatic plane-wave of the form

$$
\begin{aligned}
\mathscr{A}^{\mu}(x) & =a_{1}^{\mu} \cos (\varkappa x)+a_{2}^{\mu} \sin (\varkappa x) \\
\varkappa & =\left(\varkappa^{0}, \varkappa\right), \quad \varkappa a_{i}=0, \quad \varkappa^{2}=0 \quad \text { and } a_{1}^{2}=a_{2}^{2} \equiv a^{2}
\end{aligned}
$$

the polarization tensor splits into elastic and inelastic terms:

$$
\begin{aligned}
\Pi^{\mu \nu}\left(k, k^{\prime}\right) & =\delta_{k, k^{\prime}} \Pi_{0}^{\mu \nu}\left(k^{\prime}\right)+\delta_{k, k^{\prime}+2 \varkappa} \Pi_{-}^{\mu \nu}\left(k^{\prime}\right)+\delta_{k, k^{\prime}-2 \varkappa} \Pi_{+}^{\mu \nu}\left(k^{\prime}\right), \\
\Pi_{0}^{\mu \nu}\left(k^{\prime}\right) & =-\sum_{i= \pm, \|} \pi_{i}\left(k^{\prime}\right) \Lambda_{i}^{\mu}\left(k^{\prime}\right) \Lambda_{i}^{\nu *}\left(k^{\prime}\right), \quad \Pi_{ \pm}^{\mu \nu}\left(k^{\prime}\right)=2 \pi_{0}\left(k^{\prime}\right) \Lambda_{ \pm}^{\mu}\left(k^{\prime}\right) \Lambda_{ \pm}^{\nu}\left(k^{\prime}\right)
\end{aligned}
$$

out of which the elastic contribution $\Pi_{0}^{\mu \nu}\left(k^{\prime}\right)$ is diagonalizable. Its eigenvalues $\pi_{i}$, as well as the form factor $\pi_{0}$, are functions which have been evaluated thoroughly for the case of spinor and scalar QED in [68]. In contrast to $\Pi_{0}^{\mu \nu}\left(k^{\prime}\right)$, the other two terms in eq. (2.3) describe inelastic processes characterized by the emission or absorption of photons of the high-intensity laser wave. The involved eigenvectors $\Lambda_{+}\left(k^{\prime}\right), \Lambda_{-}\left(k^{\prime}\right)$ and $\Lambda_{\|}\left(k^{\prime}\right)$ are transverse $k^{\prime} \cdot \Lambda_{j}\left(k^{\prime}\right)=0$, orthogonal to each other $-\Lambda_{i}^{\prime}\left(k^{\prime}\right) \cdot \Lambda_{j}\left(k^{\prime}\right)=-\delta_{i j}$, and fulfill the completeness relation

$$
g^{\mu \nu}-\frac{k^{\prime \mu} k^{\prime \nu}}{k^{2}}=-\sum_{i= \pm, \|} \Lambda_{i}^{\mu}\left(k^{\prime}\right) \Lambda_{i}^{\nu}\left(k^{\prime}\right) .
$$

Particularly, we have that $\Lambda_{ \pm}$turn out to be eigenstates of opposite helicities with $\Lambda_{ \pm}^{*}=\Lambda_{\mp}$.

In its simple version, a scenario involving MCPs characterized by a mass $m_{\epsilon}$ and a tiny fraction of the electron charge $q_{\epsilon} \equiv \epsilon|e|$ is reminiscent of QED; the phenomenological consequences associated with their existence would not differ qualitatively from those emerging in a pure QED context. As such, one can investigate the related processes from already known QED expressions, with the electron parameters $(e, m)$ replaced by the respective quantities associated with a $\operatorname{MCP}\left(q_{\epsilon}, m_{\epsilon}\right)$. So, in the following, we evaluate the extent to which MCPs might influence the propagation of a probe photon in the field of the strong laser wave [eq. (2.2)] through the dispersion laws that result from the poles of the photon Green's function $\mathscr{D}_{\mu \nu}\left(k, k^{\prime}\right)$. The latter can be obtained by inversion of the two-point irreducible function [eq. (2.1)], since

$$
\int \frac{d^{4} k^{\prime \prime}}{(2 \pi)^{4}} \mathscr{D}_{\mu \rho}^{-1}\left(k, k^{\prime \prime}\right) \mathscr{D}^{\rho \nu}\left(k^{\prime \prime}, k^{\prime}\right)=-\delta_{\mu}^{\nu} \delta_{k, k^{\prime}}
$$

Indeed, by inserting the decomposition of the polarization tensor we find that - up to an inessential longitudinal contribution - the photon Green function in the field of the wave [eq. (2.2)] is given by

$$
\begin{aligned}
\mathscr{D}^{\mu \nu}\left(k, k^{\prime}\right) & =\delta_{k, k^{\prime}} \sum_{j= \pm, \|} \mathscr{D}_{j}^{\mathrm{el}} \Lambda_{j}^{\mu} \Lambda_{j}^{\nu *}+\sum_{j= \pm} \delta_{k, k^{\prime}-2 j \varkappa} \mathscr{D}_{j}^{\mathrm{in}} \Lambda_{j}^{\mu} \Lambda_{j}^{\nu} \\
\mathscr{D}_{ \pm}^{\mathrm{el}} & =-\frac{k_{ \pm}^{2}-\pi_{\mp}\left(k_{ \pm}\right)}{\left[k^{2}-\pi_{ \pm}(k)\right]\left[k_{ \pm}^{2}-\pi_{\mp}\left(k_{ \pm}\right)\right]-4 \pi_{0}(k) \pi_{0}\left(k_{ \pm}\right)}, \\
\mathscr{D}_{\|}^{\mathrm{el}} & =-\frac{1}{k^{2}-\pi_{\|}(k)}, \quad \mathscr{D}_{ \pm}^{\mathrm{in}}=-\frac{2 \pi_{0}\left(k_{ \pm}\right)}{\left[k^{2}-\pi_{ \pm}(k)\right]\left[k_{ \pm}^{2}-\pi_{\mp}\left(k_{ \pm}\right)\right]-4 \pi_{0}(k) \pi_{0}\left(k_{ \pm}\right)},
\end{aligned}
$$


where $k_{ \pm} \equiv k \pm 2 \varkappa$ and $k=(w, k)$. We remark that, in deriving the Green's function the completeness relation [eq. (2.4)] has been taken into account.

Hereafter we consider the limiting case in which the polarization effects due to MCPs are tiny corrections to the free photon dispersion equation $\left[k^{2} \simeq 0\right]$. In this approximation, the pole associated with the $\|$-mode does not correspond to photon type excitations, since — independently of the $\pi_{\|}$-structure - the corresponding eigenvector $\Lambda_{\|}$becomes purely longitudinal at $k^{2}=0$ [more details can be found in page 7 of part I of this series]. Conversely, the dispersion equations resulting from the poles associated with the transverse modes $\Lambda_{ \pm}$coincide with those found previously in refs. [66-68]:

$$
\left(k^{2}-\pi_{ \pm}(k)\right)\left[k_{ \pm}^{2}-\pi_{\mp}\left(k_{ \pm}\right)\right]=4 \pi_{0}(k) \pi_{0}\left(k_{ \pm}\right) .
$$

The corresponding vacuum refractive indices $n_{ \pm}^{2}(w, k)=k^{2} / w^{2}=1-k^{2} / w^{2}$ turn out to be

$$
n_{ \pm}^{2}(w, k)=1-\frac{\pi_{ \pm}(k)}{w^{2}}-\frac{4 \pi_{0}(k) \pi_{0}(k \pm 2 \varkappa)}{w^{2}\left[(k \pm 2 \varkappa)^{2}-\pi_{\mp}(k \pm 2 \varkappa)\right]} .
$$

The last term in the right-hand side of eq. (2.7) is responsible for inelastic transitions between states with different helicities. In the limit of interest $\left[k^{2} \simeq 0\right]$ this formula reduces to

$$
n_{ \pm}(\boldsymbol{k}) \simeq 1-\frac{\pi_{ \pm}(k)}{2 \omega_{\boldsymbol{k}}^{2}} \mp \frac{\pi_{0}(k) \pi_{0}\left(k_{ \pm}\right)}{2 \omega_{\boldsymbol{k}}^{2} k \varkappa},
$$

where $\omega_{\boldsymbol{k}} \equiv|\boldsymbol{k}|$ denotes the energy of the probe photons. Hereafter, we restrict $n_{ \pm}(\boldsymbol{k})$ to an accuracy up to terms $\sim \pi_{ \pm} / \omega_{\boldsymbol{k}}^{2}$ so that the effects resulting from the last contribution in eq. (2.8) are no longer considered. Note that this approximation is valid as long as the condition

$$
\frac{\pi_{0}\left(k_{ \pm}\right)}{\omega_{\boldsymbol{k}} \varkappa_{0}} \frac{\pi_{0}(k)}{\pi_{ \pm}(k)} \ll 1-\cos (\theta)
$$

is satisfied; otherwise the use of our perturbative treatment would not be justified. We remark that, in this expression, $\theta$ denotes the collision angle between the probe and the strong laser wave. For the particular situation to be studied later on, i.e., counterpropagating geometry $[\theta=\pi]$ with $\omega_{\boldsymbol{k}} \sim \varkappa_{0} \sim 1 \mathrm{eV}$, the above condition would imply that $\pi_{0}\left(k_{ \pm}\right) \pi_{0}(k) / \pi_{ \pm}(k) \ll 2 \mathrm{eV}^{2}$ which can be satisfied easily since the left-hand side is proportional to the square of the - presumably very tiny - coupling constant $\sim \epsilon^{2} e^{2}$. Besides, we will deal with laser waves whose intensity parameters $\xi^{2}=-e^{2} a^{2} / m^{2}$ [with $m$ and $e$ the electron mass and charge, respectively] are smaller than unity.

\subsection{Optical observables: including the paraphoton interplay}

The $\Pi_{0}^{\mu \nu}$-eigenvalues contain real and imaginary contributions $\pi_{ \pm}=\operatorname{Re} \pi_{ \pm}+i \operatorname{Im} \pi_{ \pm}$. The respective refractive indices - eq. (2.8) limited to the first two terms in the righthand side - must also be complex quantities, i.e., $n_{ \pm}=n_{ \pm}+i \varphi_{ \pm}$. While the real part $n_{ \pm}$describes the pure dispersive phenomenon, the imaginary contribution provides the absorption coefficient $\kappa_{ \pm}=\varphi_{ \pm} \omega_{\boldsymbol{k}}$ for mode- \pm photons. Accordingly, we find in the limit under consideration that

$$
n_{ \pm}=1-\left.\frac{\operatorname{Re} \pi_{ \pm}}{2 \omega_{k}^{2}}\right|_{k^{2}=0} \quad \text { and } \quad \kappa_{ \pm}=-\left.\frac{\operatorname{Im} \pi_{ \pm}}{2 \omega_{k}}\right|_{k^{2}=0} .
$$


Since the analytic properties of $\operatorname{Re} \pi_{+}$and $\operatorname{Re} \pi_{-}$are different, the vacuum behaves like a chiral birefringent medium. It is such a property which promotes the search of MCPs through the corresponding experimental measurements. Because of this, we propose a setup in which an intense circularly polarized laser pulse collides head-on with an incoming linearly polarized probe beam. With this geometry it is guaranteed that the probe beam experiences the vacuum polarization effects efficiently during the interaction time. We remark that the vacuum polarized by the strong field of the wave does not share the symmetry properties of the empty vacuum $[69,75]$. Hence, as soon as the interaction takes place, the probe decomposes accordingly into its right and left circular-polarized eigenmodes and each of them propagates with different dispersion laws as can be deduced from the respective refractive indices $\left[w_{ \pm}=|\boldsymbol{k}| / n_{ \pm}\right]$. As a consequence of this effect, the polarization of the outgoing probe is rotated by a tiny angle with respect to the incoming one:

$$
\left|\vartheta\left(\epsilon, m_{\epsilon}\right)\right| \approx \frac{1}{2}\left|\left(n_{+}-n_{-}\right) \omega_{\boldsymbol{k}} \tau\right| \ll 1,
$$

where $\tau$ is the temporal pulse length. Besides, in the field of the laser wave the vacuum is predicted to be dichroic. This effect induces a tiny ellipticity $\psi\left(\epsilon, m_{\epsilon}\right)$ in the polarization of the probe beam which is determined by the nontrivial difference between the absorption coefficients

$$
\left|\psi\left(\epsilon, m_{\epsilon}\right)\right| \approx \frac{1}{2}\left|\left(\kappa_{+}-\kappa_{-}\right) \tau\right| \ll 1 .
$$

The difference between $\kappa_{+}$and $\kappa_{-}$manifests by itself that the photo-production rate of a MCPs pair associated with a $\Pi_{0}^{\mu \nu}$-eigenwave differs from the rate resulting from the remaining mode. This statement is somewhat expected because the optical theorem dictates that the creation rate of a pair from a probe photon with polarization vector $\Lambda_{ \pm}$is given by $\mathcal{R}_{ \pm}=\Lambda_{ \pm}^{* \mu} \Lambda_{ \pm}^{\nu} \operatorname{Im} \Pi_{0 \mu \nu} / \omega=2 \kappa_{ \pm}$. We recall that the energy-momentum balance of this process $k+n \varkappa \rightarrow q_{\epsilon}^{+}+q_{\epsilon}^{-}$allows us to establish the threshold condition $n \geqslant n_{*}$, where $n_{*}=2 m_{\epsilon}^{2}\left(1+\xi_{\epsilon}^{2}\right) /(k \varkappa)$ depends on the parameter $\xi_{\epsilon}^{2}=-\epsilon^{2} e^{2} a^{2} / m_{\epsilon}^{2}$. In term of the MCP mass $m_{\epsilon}$, the previous relation translates into $m_{\epsilon} \leqslant m_{n}$, with $m_{n}$ refering to the threshold mass

$$
m_{n} \equiv \sqrt{\frac{1}{2} n k \varkappa-\epsilon^{2} m^{2} \xi^{2}} .
$$

It is worth pointing out that - as generating mechanisms of the rotation and ellipticity - the roles of the dispersion and absorption of probe photons in the field of a circularly polarized wave are exchanged with respect to the situation where a static magnetic field drives the vacuum polarization. This fact is also attributable to the different symmetries that remain in each external field configuration. For example, the symmetry group down to which the Poincaré group is broken due to the presence of an external magnetic field $\boldsymbol{B}$ is the direct product of two groups: one isomorphic to the Euclidean group $\operatorname{ISO}(2)$ and one isomorphic to the pseudo-Euclidean group $\operatorname{ISO}(1,1)[29]$. Because of this - in a magnetized vacuum - the probe beam turns out to be characterized by two propagating modes with mutually orthogonal linear polarization. Such polarization states lie on the transverse and pseudoparallel planes with respect to $\boldsymbol{B}$, i.e., where the groups $\operatorname{ISO}(2)$ and $\operatorname{ISO}(1,1)$ act, respectively. In such a background, a difference between the respective 
absorption coefficients leads to a rotated polarization direction. In contrast, the vacuum symmetry group in a circularly polarized monochromatic plane wave [eq. (2.2)] is defined from certain transformations which decompose into rotations in the plane perpendicular to the propagation and translations which compensate the rotation of the field $[69,75]$. Certainly, in this case the invariance properties of the vacuum differ from the previous case and, in correspondence, the physical modes do not have to be linearly polarized. Rather, as we already argued, these turn out to be circularly polarized and if one of two circularly polarized probe modes is less heavily damped than the other, the resulting outgoing probe field attains a tiny ellipticity which remains from this stronger mode. These two situations resemble the two known cases of birefringence and dichroism occurring in crystals [for details see [76] and references therein].

The model described so far relies on a hypothetical existence of MCPs only. Their occurrence is nevertheless naturally realized in scenarios involving hidden sectors containing an extra $\mathrm{U}(1)$ gauge group. The corresponding hidden-photon field $w_{\mu}(x)$ is massive with mass $m_{\gamma^{\prime}}$ and couples to the visible electromagnetic sector via a kinetic mixing characterized by an unknown parameter $\chi$. The diagonalization of this mixing term induces an effective interaction between the hidden-current $j_{h}^{\mu}(x)$ and the total electromagnetic field $a_{\mu}(x)+\mathscr{A}(x):$

$$
\mathcal{L}_{\mathrm{int}}=-\chi e_{h} j_{h}^{\mu}(x)\left\{a_{\mu}(x)+\mathscr{A}_{\mu}(x)\right\},
$$

where $e_{h}$ refers to the hidden gauge coupling. In addition, a mass $m_{\gamma}=\chi m_{\gamma^{\prime}}$ for the visible electromagnetic field $a_{\mu}(x)$ results. Furthermore, as a consequence of eq. (2.14), the relation $\epsilon e=-\chi e_{h}$ is established and the two-point irreducible function in the one-loop approximation becomes

$$
\mathcal{D}^{-1}\left(k, k^{\prime}\right)=-\frac{1}{4 \pi}\left[\begin{array}{cc}
\left(k^{2}-\chi^{2} m_{\gamma^{\prime}}^{2}\right) g_{\mu \nu} \delta_{k, k^{\prime}}-\Pi_{\mu \nu}\left(k, k^{\prime}\right) & \chi m_{\gamma^{\prime}}^{2} g_{\mu \nu} \delta_{k, k^{\prime}}+\frac{1}{\chi} \Pi_{\mu \nu}\left(k, k^{\prime}\right) \\
\chi m_{\gamma^{\prime}}^{2} \boldsymbol{g}_{\mu \nu} \delta_{k, k^{\prime}}+\frac{1}{\chi} \Pi_{\mu \nu}\left(k, k^{\prime}\right) & \left(k^{2}-m_{\gamma^{\prime}}^{2}\right) g_{\mu \nu} \delta_{k, k^{\prime}}-\frac{1}{\chi^{2}} \Pi_{\mu \nu}\left(k, k^{\prime}\right)
\end{array}\right] .
$$

Theoretical studies, as well as the experimental evidence indicate that the mixing parameter is much smaller than unity $[\chi \ll 1]$ so that a perturbative treatment in $\chi$ is well suited. With such an approximation, the mass term of the electromagnetic field can be ignored, leading to describe the probe photon beam by two transverse polarization states $\Lambda_{ \pm}$, whereas the $\Lambda_{\|}$-mode remains longitudinal and unphysical. ${ }^{4}$

Observe that the off-diagonal terms in $\boldsymbol{D}^{-1}\left(k, k^{\prime}\right)$ allow for the photon-paraphoton oscillation, a process driven by both: the massive terms $\chi m_{\gamma^{\prime}}^{2} g_{\mu \nu} \delta_{k, k^{\prime}}$ and those involving the vacuum polarization tensor $\frac{1}{\chi} \Pi_{\mu \nu}\left(k, k^{\prime}\right)$. However, hereafter we will suppose that the energy scale provided by the loop is much greater than the scale associated with the paraphoton mass $\left[\chi^{2} m_{\gamma}^{2} \ll \pi_{ \pm}\right]$which only leaves room for oscillations mediated by virtual

\footnotetext{
${ }^{4}$ This fact contrasts with the phenomenology occurring in a plasma, where the polarization tensor provides a longitudinal mode due to the nontrivial interplay with the medium. Indeed, if the plasma is nonrelativistic, a photon behaves like a particle with a mass determined by the plasma frequency. Under such a condition, the oscillation rate between longitudinal modes is predicted to be larger than the corresponding one between transversal modes. The relevance of this fact in deriving constraints from solar luminosity arguments has been put foward in ref. [78].
} 


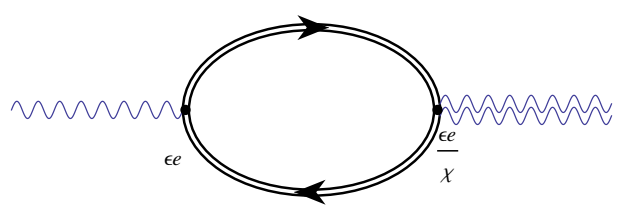

Figure 1. Pictorial representation of the one-loop photon-paraphoton vertex. The double lines represent the propagator of MCPs including the full interaction with the external field. A single wavy line denotes the amputated leg corresponding to a small-amplitude electromagnetic wave. Conversely, a double wavy line refers to the amputated leg associated with a hidden-photon field.

pairs of MCPs [see figure 1]. As a consequence of this hypothetical phenomenon, the polarization plane of a linearly-polarized probe beam should be rotated by an angle

$$
\begin{array}{r}
\left|\vartheta\left(\epsilon, m_{\epsilon}, \chi\right)\right| \approx \frac{1}{2} \mid\left(n_{+}-n_{-}\right) \omega_{\boldsymbol{k}} \tau+\chi^{2} \sin \left(\frac{n_{+}-1}{\chi^{2}} \omega_{\boldsymbol{k}} \tau\right) \exp \left(-\frac{1}{\chi^{2}} \kappa_{+} \tau\right) \\
-\chi^{2} \sin \left(\frac{n_{-}-1}{\chi^{2}} \omega_{\boldsymbol{k}} \tau\right) \exp \left(-\frac{1}{\chi^{2}} \kappa_{-} \tau\right) \mid \ll 1 .
\end{array}
$$

Observe that the first contribution coincides with the outcome resulting from a pure MCPs model [eq. (2.11)]. Hence, those terms that depend on the unknown parameter $\chi$ are connected to the photon-paraphoton oscillations.

The scenario including the hidden-photon field manifests vacuum dichroism as well, since the decay rates for the two "visible" $\Pi_{0}^{\mu \nu}$-eigenmodes, via the production of a MCPs pair and its conversion into a hidden-photon, differ from each other. The predicted ellipticity is determined by the difference between the attenuation coefficients of the propagating modes. Explicitly,

$$
\begin{array}{r}
\left|\psi\left(\epsilon, m_{\epsilon}, \chi\right)\right| \approx \frac{1}{2} \mid\left(\kappa_{-}-\kappa_{+}\right) \tau+\chi^{2} \cos \left(\frac{n_{+}-1}{\chi^{2}} \omega_{\boldsymbol{k}} \tau\right) \exp \left(-\frac{1}{\chi^{2}} \kappa_{+} \tau\right) \\
-\chi^{2} \cos \left(\frac{n_{-}-1}{\chi^{2}} \omega_{\boldsymbol{k}} \tau\right) \exp \left(-\frac{1}{\chi^{2}} \kappa_{-} \tau\right) \mid \ll 1 .
\end{array}
$$

Note that in the absence of the kinetic mixing $[\chi \rightarrow 0]$ this expression reduces to eq. (2.12). Throughout our investigation, comparisons between the pure MCPs model and the scenario including the paraphotons will be presented.

\subsection{Absorption coefficients and refractive indices at $\xi_{\epsilon}<1$}

In contrast to part I of this series, here we analyse the effects resulting from a model in which MCPs are scalar bosons. In the first place, the absence of a spin in these hypothetical degrees of freedom is manifest in the eigenvalues:

$$
\pi_{ \pm}\left(n_{*}, \xi_{\epsilon}\right)=\frac{\alpha_{\epsilon}}{2 \pi} m_{\epsilon}^{2} \int_{-1}^{1} d v \int_{0}^{\infty} \frac{d \rho}{\rho} \Omega_{ \pm} \exp \left\{-\frac{2 i \rho n_{*}}{\left(1+\xi_{\epsilon}^{2}\right)\left(1-v^{2}\right)}\left[1+2 A \xi_{\epsilon}^{2}\right]\right\} .
$$

In this expression, $\alpha_{\epsilon} \equiv \epsilon^{2} e^{2}=\epsilon^{2} / 137$ refers to the fine structure constant relative to the MCPs [with $\epsilon$ being the potentially small coupling strength in units of the absolute 
value of the electron charge $|e|]$. The expression above depends on the threshold parameter for the photo-production of a pair of MCPs $n_{*}=2 m_{\epsilon}^{2}\left(1+\xi_{\epsilon}^{2}\right) /(k \varkappa)$ [see discussion above eq. (2.13)]. Here, the functions $\Omega_{ \pm}$and $A$ read

$$
\begin{aligned}
\Omega_{ \pm} & =\xi_{\epsilon}^{2}\left[\sin ^{2}(\rho) \pm 2 i \rho A_{0}\right]+\frac{1}{2}[1-\exp (i y)], & A & =\frac{1}{2}\left[1-\frac{\sin ^{2}(\rho)}{\rho^{2}}\right] \\
A_{0} & =\frac{1}{2}\left[\frac{\sin ^{2}(\rho)}{\rho^{2}}-\frac{\sin (2 \rho)}{2 \rho}\right], & y & =\frac{4 n_{*} \xi_{\epsilon}^{2} \rho A}{\left(1+\xi_{\epsilon}^{2}\right)\left(1-v^{2}\right)} .
\end{aligned}
$$

When integrating out $v$, a compact representation of eq. (2.17) is obtained. However, the resulting integrand involves unwieldy complex functions depending on the Hankel functions of second kind $\mathrm{H}_{\nu}^{(2)}(z)=\frac{2 i}{\pi} \exp \left[\frac{i}{2} \pi \nu\right] \int_{0}^{\infty} d t \exp [-i z \cosh (t)] \cosh (\nu t)$. Explicitly, we find

$$
\begin{aligned}
\pi_{ \pm}\left(n_{*}, \xi_{\epsilon}\right)= & \frac{1}{2} \alpha_{\epsilon} m_{\epsilon}^{2} \int_{0}^{\infty} \frac{d \rho}{\rho} \Upsilon_{ \pm} \exp (-i \eta), \\
\Upsilon_{ \pm}= & -\frac{1}{2} \eta\left[\mathrm{H}_{0}^{(2)}(\eta)+i \mathrm{H}_{1}^{(2)}(\eta)\right]\left\{1+2 \xi_{\epsilon}^{2} \sin ^{2}(\rho) \pm 4 i \xi_{\epsilon}^{2} \rho A_{0}\right\} \\
& +\frac{1}{2} \rho n_{*}\left[\mathrm{H}_{0}^{(2)}\left(\rho n_{*}\right)+i \mathrm{H}_{1}^{(2)}\left(\rho n_{*}\right)\right] \exp \left[\frac{2 i \xi_{\epsilon}^{2} \rho n_{*} A}{1+\xi_{\epsilon}^{2}}\right]
\end{aligned}
$$

where the parameter $\eta \equiv n_{*} \rho(1-\Delta)$ with $\Delta=\frac{\xi_{\epsilon}^{2}}{\left(1+\xi_{\epsilon}^{2}\right)} \frac{\sin ^{2}(\rho)}{\rho^{2}}$ has been introduced.

As in I, our attention will be focused on the limit $\xi_{\epsilon}<1$. Particularly, on the simple cases in which one or two photons from the strong wave $[n=1,2]$ are absorbed. We will consider these two situations only because - for $\xi_{\epsilon}<1$ - the chiral birefringence and dichroism properties of the vacuum are predicted to be considerably more pronounced near the lowest thresholds than in the cases asymptotically far from it $\left[n_{*} \rightarrow \infty\right.$ and $\left.n_{*} \rightarrow 0\right]$, where the vacuum behaves like a nonabsorbing isotropic medium [66]. Note that in the region of interest $\left[\xi_{\epsilon}<1\right]$, the parameter $\Delta$ is much smaller than unity. So, we may Taylor expand the integrands in eqs. (2.19) - (2.20) up to second order in $\Delta$ and integrate out the $\rho$-variable. The real parts of the resulting expressions allow us to write the absorption coefficients [eq. (2.10)] in the following form:

$$
\kappa_{ \pm} \simeq \kappa_{ \pm, 1}+\kappa_{ \pm, 2}
$$

Here $\kappa_{ \pm, 1}$ and $\kappa_{ \pm, 2}$ turn out to be discontinuous contributions at the threshold point $n_{*}=1$ and $n_{*}=2$, respectively. Particularly, we find

$$
\begin{aligned}
& \kappa_{+, 1}=\frac{\alpha_{\epsilon} m_{\epsilon}^{2} \xi_{\epsilon}^{2}}{8 \omega_{k}}\left\{v_{1} \frac{1-v_{1}^{2}}{1+\xi_{\epsilon}^{2}}+\left[1-v_{1}^{2}-\frac{1-v_{1}^{4}}{2\left(1+\xi_{\epsilon}^{2}\right)}\right] \ln \left(\frac{1+v_{1}}{1-v_{1}}\right)\right\} \Theta\left[v_{1}^{2}\right], \\
& \kappa_{-, 1}=\frac{\alpha_{\epsilon} m_{\epsilon}^{2} \xi_{\epsilon}^{2}}{8 \omega_{k}}\left\{v_{1}\left(2+\frac{1-v_{1}^{2}}{1+\xi_{\epsilon}^{2}}\right)-\left[1-v_{1}^{2}+\frac{1-v_{1}^{4}}{2\left(1+\xi_{\epsilon}^{2}\right)}\right] \ln \left(\frac{1+v_{1}}{1-v_{1}}\right)\right\} \Theta\left[v_{1}^{2}\right],
\end{aligned}
$$

where $\Theta[x]$ denotes the unit step function and $v_{1}=\left(1-n_{*}\right)^{1 / 2}$ determines the relative speed between the final particles $\left|\boldsymbol{v}_{\text {rel }}\right|=2 v_{1}$ when only one photon of the intense laser wave is absorbed. We emphasize that eqs. (2.22)-(2.23) provide nonvanishing contributions whenever the MCP mass $m_{\epsilon}$ is smaller or equal to the first threshold mass $m_{1}=\left(k \varkappa / 2-\epsilon^{2} m^{2} \xi^{2}\right)^{1 / 2}$, 
corresponding to $n_{*} \leqslant 1$. Conversely, the contributions resulting from the absorption of two photons of the laser wave is valid for masses $m_{\epsilon}<m_{2}=\left(k \varkappa-\epsilon^{2} m^{2} \xi^{2}\right)^{1 / 2}$. They amount to

$$
\kappa_{ \pm, 2}=\frac{\alpha_{\epsilon} m_{\epsilon}^{2} \xi_{\epsilon}^{4}}{4 \omega_{\boldsymbol{k}}\left(1+\xi_{\epsilon}^{2}\right)}\left[\mathcal{F}_{1}\left(v_{2}\right)+2 \frac{1-v_{2}^{2}}{1+\xi_{\epsilon}^{2}} \mathcal{F}_{2}\left(v_{2}\right) \pm \mathcal{F}_{3}\left(v_{2}\right)\right] \Theta\left[v_{2}^{2}\right]
$$

where $v_{2}=\left(1-n_{*} / 2\right)^{1 / 2}$ and the functions $\mathcal{F}_{i}\left(v_{2}\right)$ with $i=1,2,3$ are given by

$$
\begin{aligned}
& \mathcal{F}_{1}\left(v_{2}\right)=v_{2}\left(1+v_{2}^{2}\right)-\left(1-v_{2}^{2}\right)^{2} \operatorname{arctanh}\left(v_{2}\right) \\
& \mathcal{F}_{2}\left(v_{2}\right)=\frac{1}{12} v_{2}\left(15 v_{2}^{4}-4 v_{2}^{2}-3\right)+\frac{1}{4}\left(1+v_{2}^{2}+3 v_{2}^{4}-5 v_{2}^{6}\right) \operatorname{arctanh}\left(v_{2}\right) \\
& \mathcal{F}_{3}\left(v_{2}\right)=\frac{1}{4} v_{2}\left(1+3 v_{2}^{2}\right)-\frac{1}{4}\left(1+3 v_{2}^{4}\right) \operatorname{arctanh}\left(v_{2}\right)
\end{aligned}
$$

Some comments are in order. Firstly, eqs. (2.24)-(2.27) were determinated by restricting the threshold parameter to $1<n_{*} \leq 2$, so that the next-to-leading order contribution $\left[\sim \xi_{\epsilon}^{4}\right]$ to the two-photon reaction is not considered. We remark that, when the scalar MCPs are created in the center-of-mass frame almost at rest $\left[v_{2} \sim 0\right.$ corresponding to $n_{*} \rightarrow 2$ ], the functions $\mathcal{F}_{i}\left(\tau_{2}\right)$ are dominated by the cubic dependences on $v_{2}$. As a consequence, the absorption coefficients for the scalar theory approach to $\kappa_{ \pm, 2} \approx \alpha_{\epsilon} m_{\epsilon}^{2} \xi_{\epsilon}^{4} v_{2}^{3}(8 \mp 1) /\left[12 \omega_{\boldsymbol{k}}\left(1+\xi_{\epsilon}^{2}\right)\right]$. Conversely, when $n_{*} \rightarrow 1$, i.e., $\left[v_{2} \rightarrow 1 / \sqrt{2}\right]$, we find the asymptotes $\kappa_{ \pm, 2} \approx \alpha_{\epsilon} m_{\epsilon}^{2} \xi_{\epsilon}^{4}(0.4 \mp 0.1) /\left[4 \omega_{k}\right]$, provided the condition $\xi_{\epsilon} \ll 1$ holds. The corresponding expression for $\kappa_{ \pm, 1}$ was derived previously in ref. [66].

In contrast to Re $\pi_{ \pm}$, the imaginary parts of $\pi_{ \pm}$are continuous functions. Hence, we only need to consider the refractive indices [eq. (2.10)] resulting from the leading term in the $\Delta$-expansion of the respective integrands [see eqs. (2.19)-(2.20)] in order to investigate the dispersion effects in the region encompassed by eqs. $(2.21)-(2.27)$, i.e., $0<n_{*} \leq 2$. After some manipulations, we end up with an integral representation for $n_{ \pm}-1$, suitable to carry out the forthcoming numerical analysis

$$
\begin{aligned}
n_{ \pm}-1 \simeq \mp \frac{\alpha_{\epsilon} m_{\epsilon}^{2} \xi_{\epsilon}^{2}}{4 \pi \omega_{k}^{2}} \int_{0}^{1} d v\{ & {\left[1 \pm \frac{2 \varrho}{1+\xi_{\epsilon}^{2}}\right] \ln \left(\frac{1+\varrho}{|1-\varrho|}\right)^{1 / 2} } \\
\mp & {\left.\left[1 \pm 2 \varrho+\frac{2 \varrho^{2}}{1+\xi_{\epsilon}^{2}}\right] \ln \left(\frac{|\varrho|}{\sqrt{\left|1-\varrho^{2}\right|}}\right)\right\} . }
\end{aligned}
$$

In this expression, $\varrho \equiv \varrho\left(v, n_{*}\right)=n_{*}\left(1-v^{2}\right)^{-1}$ is a function of both the integration variable $v$ and the threshold parameter $n_{*}$.

\section{$3 \quad$ Experimental prospects}

\subsection{Estimating the exclusion limits}

Let us estimate the projected bounds resulting from a plausible experiment in which the rotation of the polarization plane [eqs. (2.15)] and the ellipticity of the outgoing probe beam [eq. (2.16)] are probed but none of them detected. In practice, the absence of these 
signals is understood within certain confidence levels $\psi_{\mathrm{CL} \%}, \vartheta_{\mathrm{CL} \%}$, which we take hereafter as $\sim 10^{-10} \mathrm{rad}$. We emphasize that this choice of sensitivity agrees with the experimental accuracies with which - in the optical regime - both observables can nowadays be measured [77]. Thus, in the following we present the numeric outcomes resulting from the inequalities

$$
10^{-10} \mathrm{rad}>\left|\psi\left(\epsilon, m_{\epsilon}, \chi\right)\right| \text { and } 10^{-10} \mathrm{rad}>\left|\vartheta\left(\epsilon, m_{\epsilon}, \chi\right)\right| .
$$

Some comments are in order. Firstly, the sensitivity limits found from these relations will be close to reality as the parameters of the external field [eq. (2.2)] will be chosen appropriately to the monochromatic plane-wave model. In an actual experimental setup this restriction can be met by using a long pulse of duration $\tau \gg \varkappa_{0}^{-1}$ whose waist size $w_{0}$ is much greater than its wavelength $\left[w_{0} \gg \lambda_{0}\right.$ with $\left.\lambda_{0}=2 \pi \varkappa_{0}^{-1}\right]$. In this way, a negligible contribution coming from the finite bandwidth is guaranteed. Based on the previous remarks, we find it suitable to consider the benchmark parameters associated with the nanosecond frontend of the Petawatt High-Energy Laser for heavy Ion eXperiments (PHELIX) [71], $[\tau \simeq 20 \mathrm{~ns}$, $\left.w_{0} \approx 100-150 \mu \mathrm{m}, \varkappa_{0} \simeq 1.17 \mathrm{eV}, I \simeq 10^{16} \mathrm{~W} / \mathrm{cm}^{2}, \xi \simeq 6.4 \times 10^{-2}\right]$. We also investigate the results coming from the parameters associated with the nanosecond facility of the $\operatorname{LULI}(2000)$ system [72], $\left[\tau \simeq 1.5 \mathrm{~ns}, w_{0} \sim 100 \mu \mathrm{m}, \varkappa_{0} \simeq 1.17 \mathrm{eV}, I \simeq 6 \times 10^{14} \mathrm{~W} / \mathrm{cm}^{2}\right.$, $\left.\xi \simeq 2 \times 10^{-2}\right]$. Clearly, with this second analysis we seek to evaluate the extent to which the projected bounds depend on the parameters of the external field. We note that the square of the intensity parameters associated with the described laser systems are much smaller than unity $\xi^{2} \ll 1$. In such a circumstance, the corrections to the vacuum refractive indices are dominated by a quadratic dependence on $\xi$, i.e., $n_{ \pm}-1 \propto \xi^{2}$. Likewise, the terms relevant for the absorption coefficients turn out to be $\kappa_{ \pm, 1} \propto \xi^{2}$ and $\kappa_{ \pm, 2} \propto \xi^{4}$. Hence, the observables associated with the simplest MCP scenario [eqs. (2.11) and (2.12)] acquire the dependence $|\vartheta|,|\psi| \propto m^{2} \xi^{2} \tau / \omega_{\boldsymbol{k}}$. This fact indicates that - for $\omega_{\boldsymbol{k}} \sim 1 \mathrm{eV}$ - large sensitivities can be achieved provided the pulses lengths $\tau$ are long enough as to compensate for the relative smallness of $\xi$. This idea motivates the use of the nanosecond frontends of PHELIX and LULI(2000).

Now, a suitable experimental development requires a high level of synchronization between the colliding laser waves. To guarantee this important aspect, it appears convenient to use a probe obtained from the intense wave. So, we will assume a probe beam with doubled frequency $\left[\omega_{k}=2 \varkappa_{0}=2.34 \mathrm{eV}\right]$ and an intensity much smaller than the one of the strong laser field. Finally, to maximize the polarimetric effects, we will suppose that the collision between the probe and strong wave is head-on $\left[\boldsymbol{k} \cdot \varkappa=-\omega_{\boldsymbol{k}} \varkappa_{0}\right]$.

The projected exclusion regions are summarized in figure 2 . They are shaded in purple and red for PHELIX and in blue and green for LULI. These should be trustworthy as long as the limits lie below the white and black dashed lines corresponding to $\xi_{\epsilon}=\epsilon m \xi / m_{\epsilon}=1$ for LULI and PHELIX, respectively. In this figure, the left panel shows the discovery potential associated with the pure MCP model, whereas the projected bounds including the hidden-photon effects are displayed in the right panel. The results shown in the latter were obtained by setting $\chi=\epsilon$, so that the hidden coupling constant coincides with the natural value $e_{h}=e$ [see below eq. (2.14)]..$^{5}$ This assumption allows us to compare the

\footnotetext{
${ }^{5}$ From now on it must be understood that the symbol $e$ refers to the absolute value of the electron charge.
} 

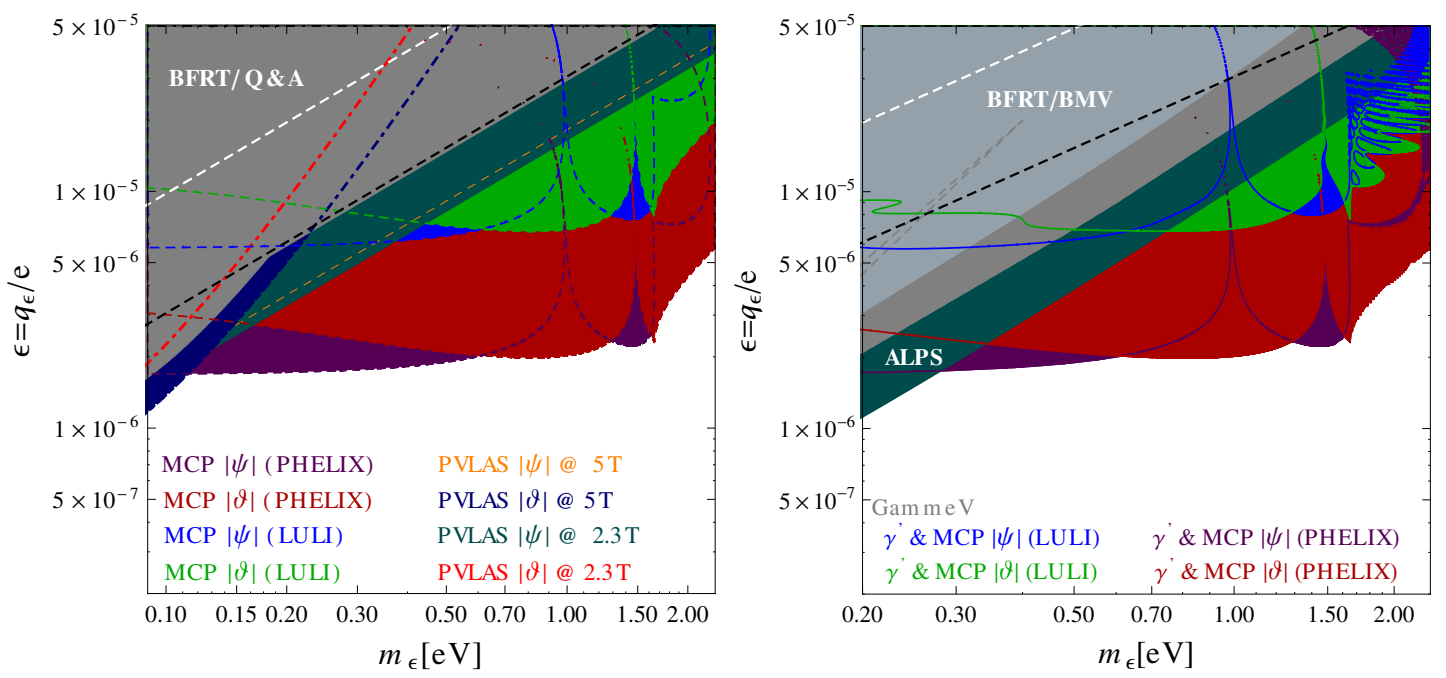

Figure 2. Estimates of constraints for MCPs of mass $m_{\epsilon}$ and relative coupling constant $\epsilon$ derived from the absence of signals in a plausible polarimetric setup assisted by a circularly polarized laser field of moderate intensity. While the left panel provides the results associated purely with MCPs, the right one shows the outcomes of the model including a hidden-photon field $\left(\gamma^{\prime}\right)$. In both panels, the white (LULI) and black (PHELIX) dashed lines correspond to the expression $\xi_{\epsilon}=1$. The left panel includes, in addition, the exclusion regions stemming from various experimental collaborations searching for rotation and ellipticity in constant magnetic fields such as BFRT [30], PVLAS [31, 32] and Q \& A [33]. The shaded areas in the upper left corner in the right panel result from experimental collaborations dealing with the Light Shining Through a Wall mechanism. The respective $95 \%$ confidence levels needed to recreate these results are summarized in ref. [12].

respective outcomes with the pure MCP model. Notice that the left panel incorporates some constraints established from other polarimetric searches [30-33]. The upper bounds which result from these experiments do not represent sensitive probes of the parameter space associated with the model containing the hidden-photon field [11]. Because of this fact, they are not displayed in the right panel. To compensate it and still put our results into perspective, we include here the limits resulting from various collaborations which deal with Light Shining Through a Wall setups [30, 37, 38, 41]. Similar to the fermion MCPs model, we observe that the most stringent sensitivity limits appear in the vicinity of the first threshold mass $m_{1} \approx 1.64 \mathrm{eV}$. This outcome follows from a search of the rotation angle. In such a situation, the projected bound turns out to be $\epsilon<2.3 \times 10^{-6}$ for PHELIX and $\epsilon<7.5 \times 10^{-6}$ for LULI. When comparing these results with the previously obtained for the model driven by fermionic MCPs $\left[\epsilon<1.9 \times 10^{-6}\right.$ for PHELIX and $\epsilon<6.5 \times 10^{-6}$ for LULI], we note that the absence of spin degrees of freedom slightly relaxes the projected sensitivity. Another interesting aspect to be highlighted in figure 2 is the curve shapes of the upper limits, which deviate from those coming out from the fermion MCPs model [cp. figure 2 in I].

Observe that, independently on whether the model includes paraphotons or not, the absence of signals for PHELIX parameters leads to similar constraints. This fact manifests 


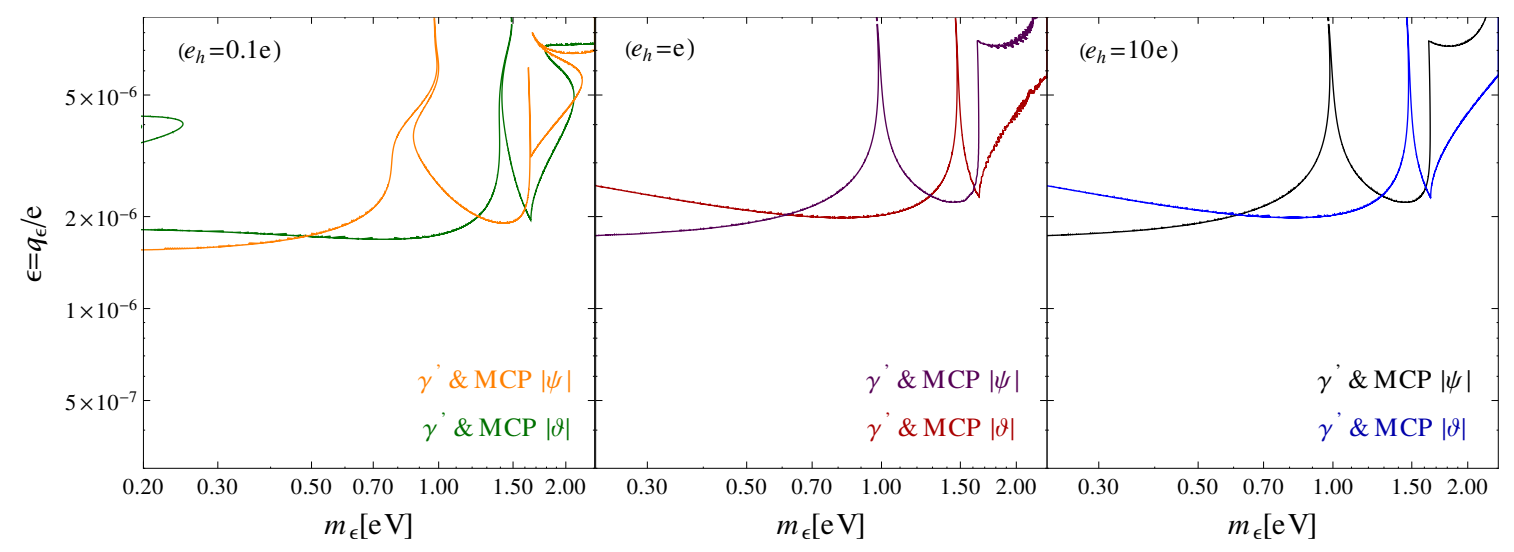

Figure 3. Parameter space to be ruled out for MCPs in a model with paraphotons $\left(\gamma^{\prime}\right)$. The expected exclusion limits have been obtained by assuming the absence of signals in a polarimetric setup assisted by a circularly polarized wave associated with the nanosecond frontend of the PHELIX laser. Here, the projected sensitivities on the kinetic mixing parameter for various values of the hidden coupling constant are displayed by contour lines [see legend].

the dominance of the first contributions to the observables in eqs. (2.15) and (2.16) for the given set of parameters. We infer that, in the region of interest within the $\left(\epsilon, m_{\epsilon}\right)$-plane, the characteristic times involved in the respective damping factors $\chi^{2} \kappa_{ \pm, 1}^{-1}$ turn out to be much smaller than the pulse lengths $\tau \gg \chi^{2} \kappa_{ \pm, 1}^{-1}$. However, the behavior is different when the LULI parameters are used. For masses in the range $m_{1}<m_{\epsilon}<m_{2}$ the respective upper bounds are characterized by an oscillatory pattern whose occurence is a direct consequence of the photon-paraphoton oscillations. This implies that, in such a regime, the characteristic times $\chi^{2} \kappa_{ \pm}^{-1}$ for LULI are much larger than the used pulse lengths $\tau$; the former being mainly determined by contributions coming from the second threshold point $\kappa_{ \pm} \simeq \kappa_{ \pm, 2}$ [see eq. (2.24)].

We continue our investigation by studying the dependence of the sensitivity limits on the hidden gauge coupling $e_{h}$. Figure 3 displays how the constraints for PHELIX might vary as $e_{h}$ changes by an order of magnitude around $e$. Taking the central panel $\left[e_{h}=e\right]$ as a reference, we note that the differences between this one and the one evaluated at $e_{h}=10 e$ [right panel] are almost imperceptible. In contrast, a notable distortion can be observed at $e_{h}=0.1 e$ [left panel]. Generally speaking, both trends resemble the results found for a spinor MCPs model. However, when directly comparing the present outcomes with those corresponding to the latter model [see figure 3 in part I of this series], we see that, at $e_{h}=0.1 e$, the absence of spin degrees of freedom strongly modifies the qualitative behavior of the projected limits. This is not the case at $e_{h}=10 e$, where the difference between the scalar and fermion models is mainly quantitative.

Perhaps the most important conclusion that one can draw from our results is that, the sensitivity limits expected for experiments driven by long laser pulses of moderate intensities would allow to discard a region of the parameter space which has not been excluded so far by other laboratory-based collaborations. Astrophysical and cosmological constraints are stronger [18-21], though, but they must be considered with some care. As 
we already mentioned in the introduction, the limits resulting from these scenarios strongly depend on models associated with certain phenomena which are not observed, such as star cooling in the first place. The vulnerability of these models has been addressed in various investigations and justifies the laboratory-based searches for these weakly interacting subeV particles [23-26]. Uncertainties introduced by parameters such as temperature, density and microscopic energy-momentum transfer are so notable that a reconciliation between the astro-cosmological constraints and those resulting from the laboratory-based experiments is achievable. To put this statement into context, let us recall that for MCPs, a study of the helium-burning phase of Horizontal-Branch (HB) stars establishes $\epsilon \leq 2 \times 10^{-14}$ for $m_{\epsilon} \lesssim \mathrm{keV}$. However, the lack of control on the physics occurring in such stellar objects might lead to the omission of suppression channels in the production of MCPs and paraphotons whose incorporation would attenuate the previous limitation. This issue has been analyzed carefully within the RM-model [24], a scenario in which two paraphotons one massless and one massive (mass $m_{\gamma^{\prime}}$ ) - are minimally coupled to dark fermions with opposite hidden charges. Owing to the incorporation of two types of paraphotons, this model turns out to be more complex than the simplest MCP scenario. We should mention however, that they can be inserted in such a way that no additional charge labeling the elementary particles is needed and leads to $\epsilon<4 \times 10^{-8}\left([\mathrm{eV}] / m_{\gamma^{\prime}}\right)^{2}$. Accordingly, less severe bounds appear when the paraphoton mass $m_{\gamma^{\prime}}$ is getting smaller. This fact fits very well with our approach since it relies on the fulfillment of the condition $m_{\gamma^{\prime}} \ll\left(\pi_{ \pm} / \chi\right)^{1 / 2}$ [see discussion above eq. (2.15)]. Note that, at the first threshold $m_{\epsilon}=m_{1}$ resulting from PHELIX parameters, $\chi<2.3 \times 10^{-6}$. So, the loop dominance in the photon-paraphoton oscillations is well justified as long as $m_{\gamma^{\prime}} \ll o[0.1-1] \mu \mathrm{eV}$, for which the constraints coming from the HB stars become much less stringent than the projected sensitivity estimated here. In part I of this series we explained that there are even certain sectors in $m_{\gamma^{\prime}}$ in which our projected upper bounds for $\chi$ turn out to be currently the best model-independent results. Similar conclusions can be drawn from a study of a hypothetical solar emission of hidden massive photons for which the constraint $\chi<4 \times 10^{-12}\left(\mathrm{eV} / m_{\gamma^{\prime}}\right)$ for $m_{\gamma^{\prime}} \lesssim 3 \mathrm{eV}$ has been established [79].

\subsection{Characteristic of the signals in the scalar MCPs model}

Suppose that the outgoing probe beam acquires an ellipticity and rotation which do not coincide with the QED prediction [cp. discussion in section 3.1 of I]. If their origin can be attributed to MCPs,${ }^{6}$ the next questions of interest are: do the signals come from the existence of scalar or spinor MCPs; and do they manifest the effects intrinsically associated with hidden-photons? The answers to these questions can be obtained by investigating the dependencies of the observables on the laser parameters. In this subsection, we provide arguments which might help to discern the phenomenological differences that result from the various MCP models of interest. Our discussion will be based on the outcomes derived from the benchmark parameters of the nanosecond frontend of PHELIX [standard values

\footnotetext{
${ }^{6}$ In first instance, these signals might not only result from the existence of MCPs and paraphotons but also from other light dark matter candidates such as axion-like particles.
} 


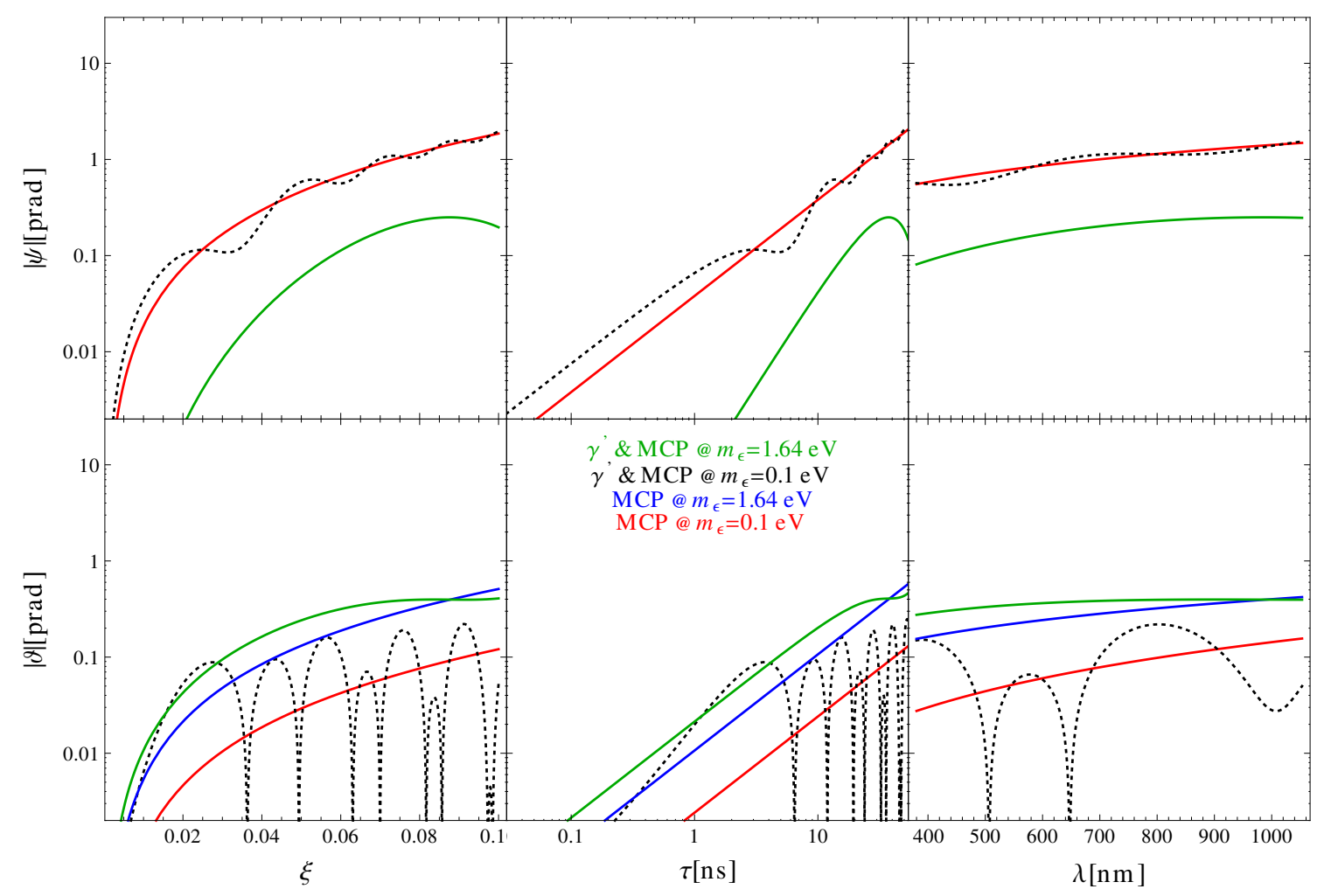

Figure 4. Dependence of the absolute value of the ellipticity $|\psi|$ [upper panels] and rotation angle $|\vartheta|$ [lower panels] on the intensity parameter $\xi$ [left panel], pulse length $\tau$ [central panel] and wavelength of the probe $\lambda=\lambda_{0} / 2$ [right panel]. As a benchmark point we assume a massless hidden photon field with kinetic mixing parameter $\chi=5 \times 10^{-7}$ and hidden coupling $e_{h}=e$. In each plot the remaining external parameters are kept at $\xi=6.4 \times 10^{-2}, \tau=20 \mathrm{~ns}, \theta=\pi$, and $\lambda_{0}=1053 \mathrm{~nm}$ the wavelength of the intense laser field. Here the outcomes resulting from a pure MCP model at $m_{\epsilon}=0.1 \mathrm{eV}$ are shown in red, whereas the respective patterns at the first threshold mass $m_{1} \approx(k \varkappa / 2)^{1 / 2}=1.64 \mathrm{eV}$ are in blue. The curves in green and dotted black were obtained by including the paraphoton field. They also correspond to the case in which the mass of the minicharges are $m_{\epsilon}=m_{1}$ and $m_{\epsilon}=0.1 \mathrm{eV}$, respectively. Observe that the blue curves corresponding to the pure MCPs model at $m_{1}=1.64 \mathrm{eV}$ - do not appear in the upper panel. This is because, at the first threshold mass, the ellipticity becomes extremely tiny being determined by the next-to-leading order term in the absorption coefficient [eqs. (2.24)-(2.27)].

$\left.\xi=6.4 \times 10^{-2}, \tau=20 \mathrm{~ns}, \lambda_{0}=1053 \mathrm{~nm}\right]$ and by considering a probe beam with $\lambda=\lambda_{0} / 2$ which collides head-on with the intense laser wave. To facilitate the comparisons between the scalar and fermion models, each figure in this subsection will encompass the same scales as used in part I of this series.

The behavior of the signals with respect to the laser intensity parameter $\xi$, the temporal length $\tau$ and the wave length of the probe beam $\lambda=2 \pi \omega^{-1}$ are displayed in figure 4 . These results have been obtained at the first threshold mass $m_{\epsilon}=m_{1} \approx(k \varkappa / 2)^{1 / 2} \approx 1.64 \mathrm{eV}$ and at $m_{\epsilon}=0.1 \mathrm{eV}$. While the outcomes associated with the pure MCP scenario are shown in blue and red, the results including the paraphoton effects are shown in green and 
black dotted curves. To facilitate our discussion, we will first focus on the behavior of the absolute value of the ellipticity $\left|\psi\left(\epsilon, m_{\epsilon}, \chi\right)\right|$. Out of the threshold, i.e. at $m_{\epsilon}=0.1 \mathrm{eV}$, this observable [upper panels] grows with the increase of the three laser parameters. The curves associated with the model including a paraphoton field [black dotted lines in the upper panel] manifest pronounced oscillatory behaviors around the paths followed by the pure MCPs framework [in red]. This fluctuating patterns were also revealed in the fermion case, although in a tiny - almost imperceptible - degree only. The occurrence of this trend at $m_{\epsilon}=0.1 \mathrm{eV}$ is closely related to the photon-paraphoton oscillation, which seems to benefit from the absence of the spin degrees of freedom. The behavior of $\left|\psi\left(\epsilon, m_{\epsilon}, \chi\right)\right|$ at the first threshold mass $\left[m_{\epsilon}=m_{1}\right]$ is different. Here the curves in green [upper panel] can be exploited to elucidate the nature of the charged carriers. To do this, we note that the oscillatory patterns in the ellipticity spread considerably as compared with those corresponding to the fermion MCPs model [see upper panel in figure 5]. As such, the displayed curves for scalar MCPs do not show oscillations within the investigated intervals for $\xi, \tau$ and $\lambda$. This fact constitutes a remarkable property because it implies that a slight variation of the intensity could not lead to change the signal sign for the scalar MCPs model, but it might change $\psi\left(\epsilon, m_{\epsilon}, \chi\right)$ substantially if it is induced by the fermion model. Clearly, this analysis is also applicable to the remaining parameters of the external laser wave.

The reason why the ellipticity curves for scalar MCPs do not change the sign can be understood as follows: at $m_{\epsilon}=m_{1}$, the charge carriers tend to be produced at rest $\left[v_{1} \rightarrow 0\right]$, so that the leading order terms in the absorption coefficients [eq. (2.21)] tend to vanish. As a consequence, the characteristic times $\sim \chi^{2} \kappa_{ \pm, 2}^{-1}$ increase and can reach values much larger than the corresponding pulse length $\tau$. Accordingly, the exponential damping factors in eq. (2.16) can approach unity. Besides, by quoting the refractive indices from ref. [66]: $\left.\left(n_{i}-1\right)\right|_{n_{*}=0} \approx-\alpha_{\epsilon} m_{1}^{2} \xi_{\epsilon}^{2} /\left(5 \pi \omega_{\boldsymbol{k}}^{2}\right) \delta_{-, i}$ with $i=+,-$ we find that the asymptotic expression for the ellipticity is determined by the oscillation probabilities between a photon and a paraphoton with negative helicities $\mathcal{P}_{\gamma_{-} \rightarrow \gamma_{-}^{\prime}}:^{7}$

$$
\psi\left(\epsilon, m_{1}, \chi\right) \approx \frac{1}{4} \mathcal{P}_{\gamma_{-} \rightarrow \gamma_{-}^{\prime}} \quad \text { with } \quad \mathcal{P}_{\gamma_{-} \rightarrow \gamma_{-}^{\prime}}=4 \chi^{2} \sin ^{2}\left(\frac{n_{-}-1}{2 \chi^{2}} \omega_{k} \tau\right) .
$$

Manifestly, in figure 5, the green curves resemble the $\sin ^{2}$-shape obtained above. We remark that, in contrast to the fermion model, the remaining oscillation probability in the scalar scenario tends to vanish identically $\left[\mathcal{P}_{\gamma_{+} \rightarrow \gamma_{+}^{\prime}} \approx 0\right] .^{8}$

A similar study allows us to find the asymptote for the absolute value of the rotation angle $\vartheta\left(\epsilon, m_{\epsilon}, \chi\right)$ at the first threshold point $\left[m_{\epsilon}=m_{1}\right]$. In this case,

$$
\vartheta\left(\epsilon, m_{1}, \chi\right) \approx-\frac{1}{2} \chi^{2}[s+\sin (s)], \quad s \equiv \frac{n_{-}-1}{\chi^{2}} \omega_{k} \tau .
$$

Observe that, since the refractive index $n_{-}-1<0$, we have $s<0$ and the involved function $s+\sin (s) \leqslant 0$. As a consequence, the rotation angle does not change the sign either, a

\footnotetext{
${ }^{7} \mathrm{~A}$ general expression for the oscillation probability between photon and paraphoton has already been derived [see eq. (2.38) in I]: $\mathcal{P}_{\gamma_{ \pm} \rightarrow \gamma_{ \pm}^{\prime}}(\tau) \simeq \chi^{2}\left\{1+\exp \left(-\frac{2}{\chi^{2}} \kappa_{ \pm} \tau\right)-2 \exp \left(-\frac{1}{\chi^{2}} \kappa_{ \pm} \tau\right) \cos \left(\frac{n_{ \pm}-1}{\chi^{2}} \omega_{\boldsymbol{k}} \tau\right)\right\}$.

${ }^{8}$ As $\tau \ll \chi^{2} \kappa_{+}^{-1}$ and $n_{+}-1 \approx 0$, it results $\mathcal{P}_{\gamma_{+} \rightarrow \gamma_{+}^{\prime}}(\tau) \approx 0$.
} 


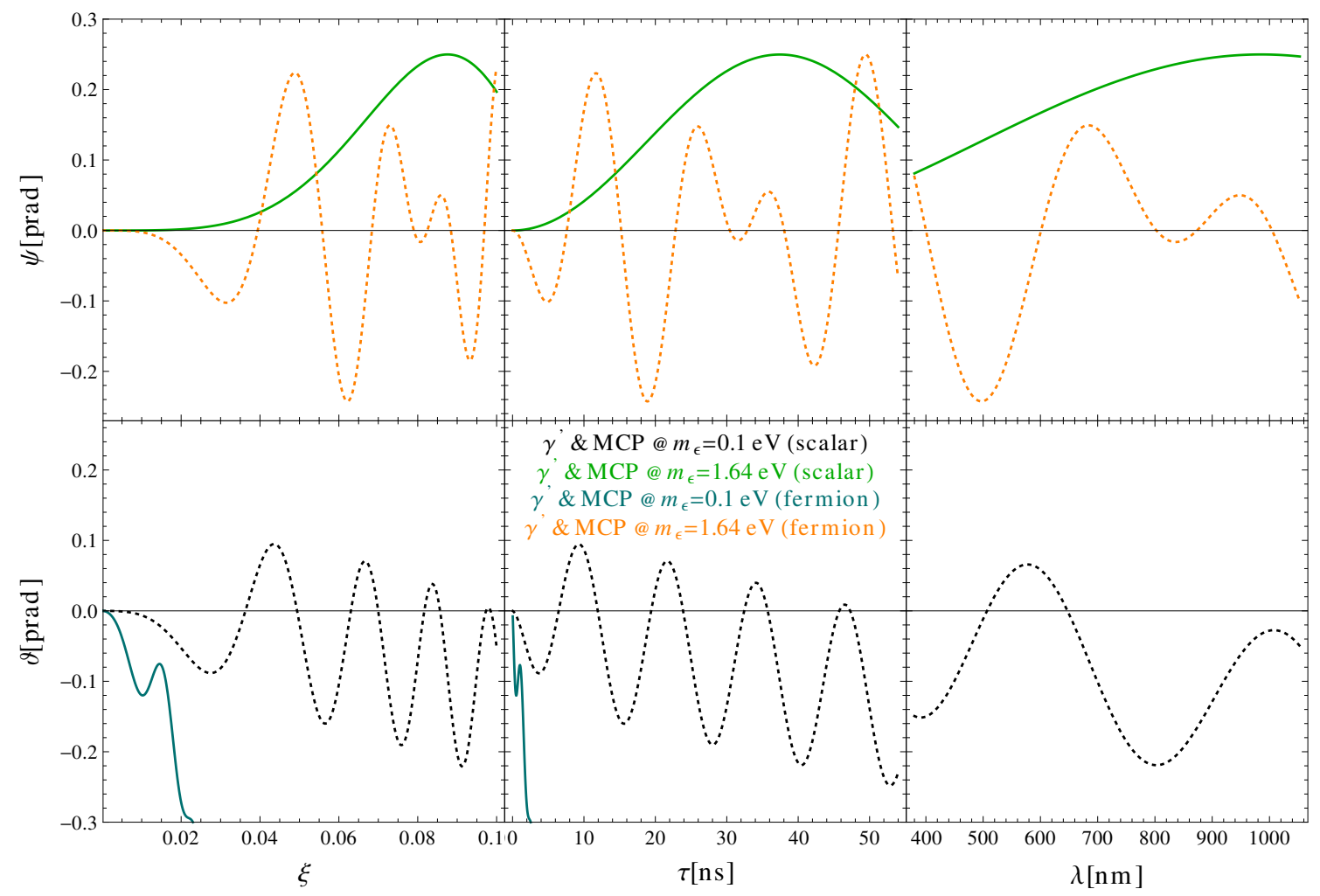

Figure 5. Behavior of the the ellipticity $\psi\left(\epsilon, m_{\epsilon}\right)$ [upper panels] and rotation angle $\vartheta\left(\epsilon, m_{\epsilon}\right)$ [lower panels] on the intensity parameter $\xi$ [left panel], pulse length $\tau$ [central panel] and wavelength of the probe $\lambda$ [right panel] in models including fermion versus scalar MCPs. The same parameter values and notation as in figure 4 are used, but the plots are in linear scales.

fact which is manifest in figure 4 [lower panel]. We note that, at the first threshold mass $\left[m_{1} \simeq 1.64 \mathrm{eV}\right]$, no manifestation of oscillations appears within the range of interest in the external field parameters. However, at $m_{\epsilon}=m_{1}$, the patterns found in the fermionic model with a hidden photon field fluctuate about the curves which result from the pure MCPs scenario. At this point we shall recall that - in contrast to the ellipticity - such oscillations for $\vartheta\left(\epsilon, m_{\epsilon}, \chi\right)$ do not change the sign [see I for details]. Therefore, if on variating $\xi, \tau$ and $\lambda$, the signal does not oscillate as described previously, then one could associate the measurements with the scalar model. Still, this way of elucidating the nature of the involved charge carriers may be considered more difficult than the approach associated with the ellipticity since no change of sign arises.

Regarding the behavior of the rotation angle at $m_{\epsilon}=0.1 \mathrm{eV}$, the occurrence of highly oscillating patterns in the model with paraphotons is notable [black dotted curves in figures 4 and 5, lower panels]. The corresponding trend associated with the fermion model turns out to be much less pronounced. While in this last scenario there is no change of sign, in the scalar case the signal might change. This is because, for the present benchmark parameters, the characteristic time associated with the negative helicity mode $\sim \chi^{2} \kappa_{-}^{-1}$ becomes much smaller than the pulse length $[\tau=20 \mathrm{~ns}]$, leading to an exponential suppression 


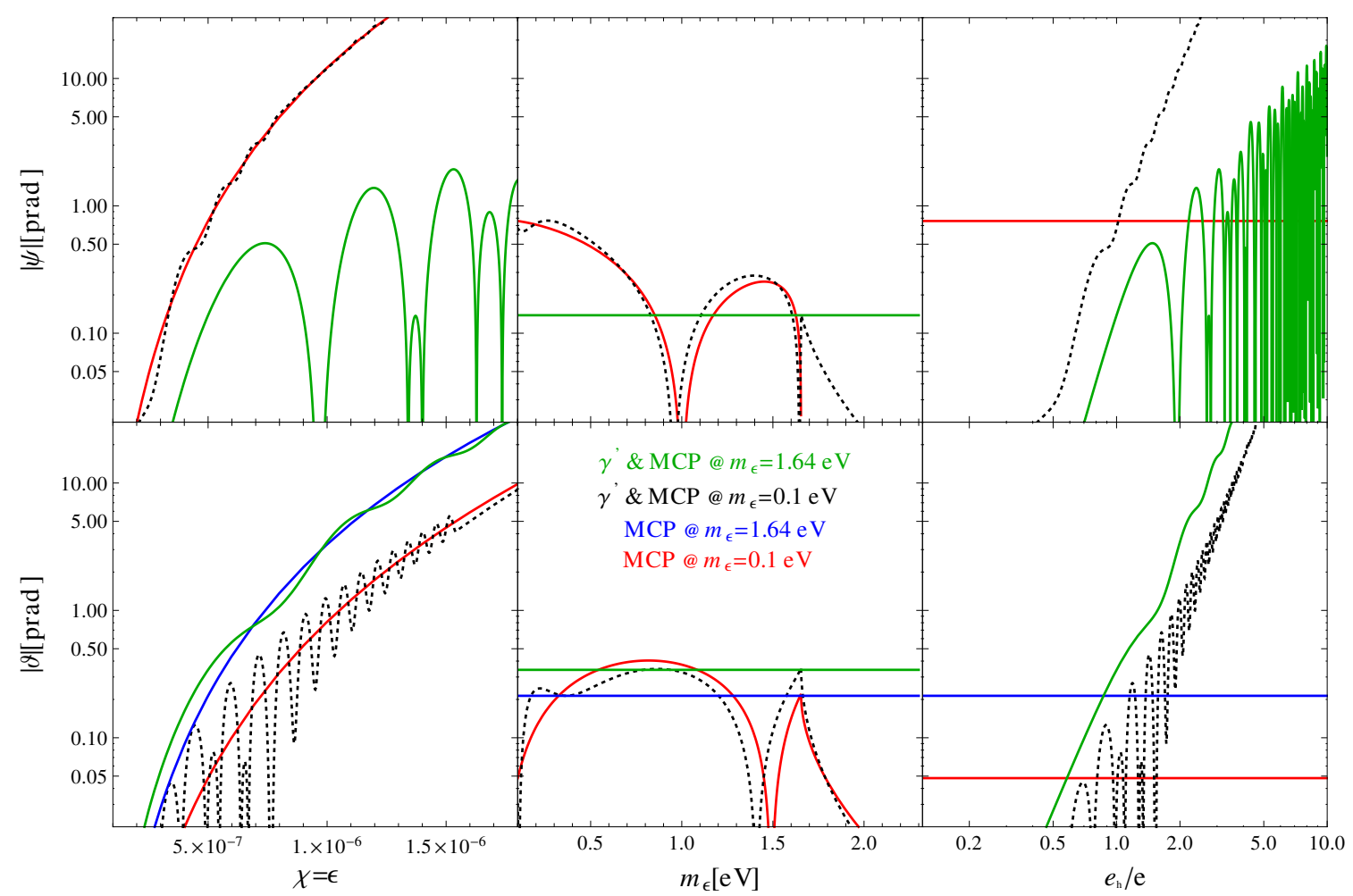

Figure 6. Dependence of the absolute value of the ellipticity $|\psi|$ [upper panels] and rotation angle $|\vartheta|$ [lower panels] on the kinetic mixing parameter $\chi$ [left panel], mass $m_{\epsilon}$ [central panel] and the relative hidden coupling $e_{h} / e$ [right panel]. The same benchmark values and notation as in figure 4 are used.

of the last term in eq. (2.15). Conversely, the characteristic time related to the positive helicity mode is $\chi^{2} \kappa_{+}^{-1} \gg \tau$. In such a situation, the remaining damping factor in eq. (2.15) can be approached by unity and

$$
\left|\vartheta\left(\epsilon, m_{\epsilon}, \chi\right)\right| \approx \frac{1}{2}\left|\left(n_{+}-n_{-}\right) \omega_{\boldsymbol{k}} \tau+\chi^{2} \sin \left(\frac{n_{+}-1}{\chi^{2}} \omega_{\boldsymbol{k}} \tau\right)\right| .
$$

Thus, as in the case of the ellipticity, one might — by changing the external field parameters - use a change of sign to elucidate whether scalar MCPs are realized or not in nature. Although eq. (3.4) looks similar to eq. (3.3) it differs from the latter in the important respect that it involves the refractive index $n_{+}-1$ which - in the current regime of mass - does not vanish identically.

Finally, in figure 6, the dependencies of the ellipticity and rotation of the polarization plane with respect to some unknown parameters are shown. The vertical central panel of this figure displays how the signals might change with the mass $m_{\epsilon}$ of this hypothetical charge carriers. As in the fermion model, the ellipticity resulting from the scenario without paraphotons reveals a discontinuity at the first threshold mass [red curve], discussed in section 2.3, which is smoothed as soon as a hidden photon field is taken into account [dotted black curve]. As a side remark, we point out that at the first threshold, the ellipticity is constant in both models. Note that the blue curves - corresponding to the pure MCPs model 
at $m_{1}=1.64 \mathrm{eV}$ - do not appear in the upper panels neither in figure 4 nor in figure 6 . This is because, at the first threshold mass, the ellipticity becomes extremely tiny being determined by the next-to-leading order term in the absorption coefficient [eqs. (2.24)(2.27)]. We note that, in contrast to the ellipticity, the dependence of $\left|\vartheta\left(\epsilon, m_{\epsilon}, \chi\right)\right|$ with respect to the mass $m_{\epsilon}$ follows a continuous paths in both models. Regarding the left and right vertical panels, they illustrate how both observables depend on the mixing parameter $\chi$ and the relative hidden coupling $e_{h} / e$. In both panels the fluctuating patterns for the ellipticity [eqs. (3.2)] and rotation of the polarization plane [eq. (3.4)], at the respective masses $m_{1}=1.64 \mathrm{eV}$ and $m_{\epsilon}=0.1 \mathrm{eV}$ can be seen. Particularly, the outcomes associated with the latter observable in the lower left panel manifest that the curve including a hiddenphoton field is modulated around the pure MCPs contribution [first term in the right-hand side of [eq. (3.4)]. Both panels show a fast decrease of the observables for small values of $\chi$, a trend which is also manifest with respect to $e_{h} / e$ [black dotted curve]. We remark that, in the right panel, the outcomes resulting from the pure MCP scenario [horizontal red and blue lines] are not sensitive to variations of the relative hidden coupling because the latter only emerges within the framework of a hidden-photon field.

\section{Conclusions and outlook}

Experiments designed to detect the QED vacuum birefringence in laser pulses might provide insights about light dark matter candidates such as MCPs and paraphotons. Throughout this investigation, we have paid special attention to the capability which long laser pulses $[\tau \sim \mathrm{ns}]$ of moderate intensities $[\xi<1]$ offer for the exploration of new domains of particle physics. Particularly, we have pointed out that their long durations compensate the small intensities associated with them and the combination of this feature with the fact that they are also characterized by a well-defined frequency manifests the realization of thresholds in which the projected sensitivities can be higher than those achieved in experiments driven by dipole magnets. We have noted that - depending on the external parameters - the absence of spin can facilitate or counteract the photon-paraphoton oscillations, as compared with the fermion MCPs model. This intrinsic property might manifest through the probe photon beam and, can be exploited to discern the quantum statistics of these particle candidates. A special emphasis has been laid on a plausible change in the ellipticity sign that the probe photon can undergo, depending upon the MCPs nature.

Finally, we emphasize that the treatment used in this investigation is valid only for $\xi_{\epsilon} \ll 1$. It would be interesting to extend the present research to the case in which $\xi_{\epsilon}>1$. We remark that, the estimated upper bounds $\left[\epsilon \sim 10^{-6}-10^{-5}\right.$ for $\left.m_{\epsilon} \sim 0.1-1 \mathrm{eV}\right]$ can lead to an intensity parameter greater than unity $\left[\xi=\frac{m_{\epsilon}}{\epsilon m} \xi_{\epsilon} \gg 1\right]$, provided $\xi_{\epsilon} \gg 1$. Corresponding laser sources exist. Indeed, intensities as large as $\sim 10^{22} \mathrm{~W} / \mathrm{cm}^{2}$ have already been achieved by the HERCULES petawatt system [50] and a substantial intensity upgrade is foreseen at ELI and XCELS [51, 52]. In connection with these high-intensity petawatt sources, the HIBEF consortium [60] has proposed an experiment to measure vacuum birefringence for the first time by combining a very intense optical pulse with $\xi \gg 1$ and a probe x-ray free electron laser [57]. Certainly, these measurements will provide a 
genuine opportunity to search for axion-like particles, MCPs and paraphotons. However, in constrast to our treatment, a theoretical description of a polarimetric experiment assisted by such pulses is complicated by the fact that - as a result of the focusing - their typical spatial extensions $d \sim \mu \mathrm{m}$ are comparable with their wavelengths. As a consequence, the monochromatic model for the external field [eq. (2.2)] is no longer valid and the pulse profile becomes relevant for the establishment of the exclusion limits. For axion-like particles a study of this nature has already been carried out [64], but it remains intriguing to see how the wave profile can influence the upper bounds associated with MCPs and hidden photon fields.

\section{Acknowledgments}

We gratefully acknowledge useful discussions with Holger Gies and funding by the German Research Foundation (DFG) under Grant No. MU 3149/2-1.

Open Access. This article is distributed under the terms of the Creative Commons Attribution License (CC-BY 4.0), which permits any use, distribution and reproduction in any medium, provided the original author(s) and source are credited.

\section{References}

[1] WMAP collaboration, G. Hinshaw et al., Nine-Year Wilkinson Microwave Anisotropy Probe (WMAP) Observations: Cosmological Parameter Results, Astrophys. J. Suppl. 208 (2013) 19 [arXiv: 1212.5226] [INSPIRE].

[2] D. Clowe et al., A direct empirical proof of the existence of dark matter, Astrophys. J. 648 (2006) L109 [astro-ph/0608407] [INSPIRE].

[3] F. Iocco, M. Pato and G. Bertone, Evidence for dark matter in the inner Milky Way, Nature Phys. 11 (2015) 245 [arXiv: 1502.03821] [INSPIRE].

[4] Planck collaboration, P.A.R. Ade et al., Planck 2015 results. XIII. Cosmological parameters, arXiv:1502.01589 [INSPIRE].

[5] E. Witten, Some Properties of O(32) Superstrings, Phys. Lett. B 149 (1984) 351 [InSPIRE].

[6] O. Lebedev, H.P. Nilles, S. Ramos-Sanchez, M. Ratz and P.K.S. Vaudrevange, Heterotic mini-landscape. (II). Completing the search for MSSM vacua in a Z(6) orbifold, Phys. Lett. B 668 (2008) 331 [arXiv:0807.4384] [INSPIRE].

[7] O. Lebedev and S. Ramos-Sanchez, The NMSSM and String Theory, Phys. Lett. B 684 (2010) 48 [arXiv: 0912.0477] [INSPIRE].

[8] M. Goodsell, J. Jaeckel, J. Redondo and A. Ringwald, Naturally Light Hidden Photons in LARGE Volume String Compactifications, JHEP 11 (2009) 027 [arXiv:0909.0515] [INSPIRE].

[9] L.B. Okun, Limits of electrodynamics: paraphotons?, Sov. Phys. JETP 56 (1982) 502 [INSPIRE].

[10] P. Langacker, The Physics of Heavy Z' Gauge Bosons, Rev. Mod. Phys. 81 (2009) 1199 [arXiv:0801.1345] [INSPIRE]. 
[11] M. Ahlers, H. Gies, J. Jaeckel, J. Redondo and A. Ringwald, Light from the hidden sector, Phys. Rev. D 76 (2007) 115005 [arXiv:0706. 2836] [inSPIRE].

[12] M. Ahlers, H. Gies, J. Jaeckel, J. Redondo and A. Ringwald, Laser experiments explore the hidden sector, Phys. Rev. D 77 (2008) 095001 [arXiv:0711.4991] [INSPIRE].

[13] B. Holdom, Two U(1)'s and Epsilon Charge Shifts, Phys. Lett. B 166 (1986) 196 [InSPIRE].

[14] B.A. Dobrescu and I. Mocioiu, Spin-dependent macroscopic forces from new particle exchange, JHEP 11 (2006) 005 [hep-ph/0605342] [INSPIRE].

[15] H. Gies, J. Jaeckel and A. Ringwald, Polarized Light Propagating in a Magnetic Field as a Probe of Millicharged Fermions, Phys. Rev. Lett. 97 (2006) 140402 [hep-ph/0607118] [INSPIRE].

[16] B. Döbrich and H. Gies, Interferometry of light propagation in pulsed fields, Europhys. Lett. 87 (2009) 21002 [arXiv: 0904.0216] [INSPIRE].

[17] H. Gies, Strong laser fields as a probe for fundamental physics, Eur. Phys. J. D 55 (2009) 311 [arXiv: 0812.0668] [INSPIRE].

[18] J. Jaeckel and A. Ringwald, The Low-Energy Frontier of Particle Physics, Ann. Rev. Nucl. Part. Sci. 60 (2010) 405 [arXiv: 1002. 0329] [INSPIRE].

[19] A. Ringwald, Exploring the Role of Axions and Other WISPs in the Dark Universe, Phys. Dark Univ. 1 (2012) 116 [arXiv:1210.5081] [INSPIRE].

[20] J.L. Hewett et al., Fundamental Physics at the Intensity Frontier, proceedings of the 2011 workshop on Fundamental Physics at the Intensity Frontier, Rockville, MD, U.S.A., November 30 - December 2 2011, arXiv:1205.2671 [INSPIRE].

[21] R. Essig et al., Working Group Report: New Light Weakly Coupled Particles, arXiv: 1311.0029 [INSPIRE].

[22] S. Davidson, S. Hannestad and G. Raffelt, Updated bounds on millicharged particles, JHEP 05 (2000) 003 [hep-ph/0001179] [INSPIRE].

[23] E. Masso and J. Redondo, Evading astrophysical constraints on axion-like particles, JCAP 09 (2005) 015 [hep-ph/0504202] [INSPIRE].

[24] E. Masso and J. Redondo, Compatibility of CAST search with axion-like interpretation of PVLAS results, Phys. Rev. Lett. 97 (2006) 151802 [hep-ph/0606163] [INSPIRE].

[25] J. Jaeckel, E. Masso, J. Redondo, A. Ringwald and F. Takahashi, We need lab experiments to look for axion-like particles, hep-ph/0605313 [INSPIRE].

[26] J. Jaeckel, E. Masso, J. Redondo, A. Ringwald and F. Takahashi, The need for purely laboratory-based axion-like particle searches, Phys. Rev. D 75 (2007) 013004 [hep-ph/0610203] [INSPIRE].

[27] W. Dittrich and H. Gies, Probing the quantum vacuum, Springer, Heidelberg, Germany (2000).

[28] K. Hattori and K. Itakura, Vacuum birefringence in strong magnetic fields: (I) Photon polarization tensor with all the Landau levels, Annals Phys. 330 (2013) 23 [arXiv: 1209.2663] [INSPIRE].

[29] S. Villalba-Chávez and A.E. Shabad, QED with external field: Hamiltonian treatment for anisotropic medium formed by the Lorentz-non-invariant vacuum, Phys. Rev. D 86 (2012) 105040 [arXiv: 1206.4491 ] [INSPIRE]. 
[30] R. Cameron et al., Search for nearly massless, weakly coupled particles by optical techniques, Phys. Rev. D 47 (1993) 3707 [INSPIRE].

[31] PVLAS collaboration, E. Zavattini et al., New PVLAS results and limits on magnetically induced optical rotation and ellipticity in vacuum, Phys. Rev. D 77 (2008) 032006 [arXiv:0706.3419] [INSPIRE].

[32] F. Della Valle et al., Measurements of vacuum magnetic birefringence using permanent dipole magnets: the PVLAS experiment, New J. Phys. 15 (2013) 053026 [arXiv:1301.4918] [INSPIRE].

[33] S.-J. Chen, H.-H. Mei and W.-T. Ni, Q $\& 3$ A experiment to search for vacuum dichroism, pseudoscalar-photon interaction and millicharged fermions, Mod. Phys. Lett. A 22 (2007) 2815 [hep-ex/0611050] [INSPIRE].

[34] R. Battesti et al., The BMV experiment: a novel apparatus to study the propagation of light in a transverse magnetic field, Eur. Phys. J. D 46 (2008) 323 [arXiv:0710.1703].

[35] J. Jaeckel, Probing Minicharged Particles with Tests of Coulomb's Law, Phys. Rev. Lett. 103 (2009) 080402 [arXiv:0904.1547] [INSPIRE].

[36] J. Jaeckel and S. Roy, Spectroscopy as a test of Coulomb's law: A probe of the hidden sector, Phys. Rev. D 82 (2010) 125020 [arXiv:1008.3536] [InSPIRE].

[37] K. Ehret et al., New ALPS Results on Hidden-Sector Lightweights, Phys. Lett. B 689 (2010) 149 [arXiv: 1004.1313] [INSPIRE].

[38] GammeV (T-969) collaboration, A.S. Chou et al., Search for axion-like particles using a variable baseline photon regeneration technique, Phys. Rev. Lett. 100 (2008) 080402 [arXiv: 0710.3783] [INSPIRE].

[39] A. Afanasev et al., New Experimental limit on Optical Photon Coupling to Neutral, Scalar Bosons, Phys. Rev. Lett. 101 (2008) 120401 [arXiv:0806.2631] [INSPIRE].

[40] OSQAR collaboration, P. Pugnat et al., First results from the OSQAR photon regeneration experiment: No light shining through a wall, Phys. Rev. D 78 (2008) 092003 [arXiv:0712.3362] [INSPIRE].

[41] C. Robilliard et al., No light shining through a wall, Phys. Rev. Lett. 99 (2007) 190403 [arXiv:0707.1296] [INSPIRE].

[42] M. Fouche et al., Search for photon oscillations into massive particles, Phys. Rev. D 78 (2008) 032013 [arXiv:0808.2800] [INSPIRE].

[43] J. Redondo and A. Ringwald, Light shining through walls, Contemp. Phys. 52 (2011) 211 [arXiv: 1011.3741] [INSPIRE].

[44] P. Arias, J. Jaeckel, J. Redondo and A. Ringwald, Optimizing Light-Shining-through-a-Wall Experiments for Axion and other WISP Searches, Phys. Rev. D 82 (2010) 115018 [arXiv: 1009.4875] [INSPIRE].

[45] B. Döbrich, H. Gies, N. Neitz and F. Karbstein, Magnetically amplified tunneling of the 3rd kind as a probe of minicharged particles, Phys. Rev. Lett. 109 (2012) 131802 [arXiv: 1203.2533] [INSPIRE].

[46] H. Gies, J. Jaeckel and A. Ringwald, Accelerator Cavities as a Probe of Millicharged Particles, Europhys. Lett. 76 (2006) 794 [hep-ph/0608238] [INSPIRE]. 
[47] R. Bähre et al., Any light particle search II - Technical Design Report, 2013 JINST 8 T09001 [arXiv: 1302.5647] [INSPIRE].

[48] P. Sikivie, D.B. Tanner and K. van Bibber, Resonantly enhanced axion-photon regeneration, Phys. Rev. Lett. 98 (2007) 172002 [hep-ph/0701198] [INSPIRE].

[49] A. Di Piazza, C. Müller, K.Z. Hatsagortsyan and C.H. Keitel, Extremely high-intensity laser interactions with fundamental quantum systems, Rev. Mod. Phys. 84 (2012) 1177 [arXiv:1111.3886] [INSPIRE].

[50] V. Yanovsky et al., Ultra-high intensity-300-TW laser at $0.1 \mathrm{~Hz}$ repetition rate, Opt. Express 16 (2008) 2109.

[51] https://eli-laser.eu/.

[52] http://www.xcels.iapras.ru/.

[53] B. King, P. Böhl and H. Ruhl, Interaction of photons traversing a slowly varying electromagnetic background, Phys. Rev. D 90 (2014) 065018 [arXiv:1406.4139] [InSPIRE].

[54] A. Di Piazza, A.I. Milstein and C.H. Keitel, Photon splitting in a laser field, Phys. Rev. A 76 (2007) 032103 [arXiv:0704.0695] [InSPIRE].

[55] B. King, A. Di Piazza and C.H. Keitel, A matterless double slit, Nature Photonics 4 (2010) 92.

[56] K. Hatsagortsyan and G.Y. Kryuchkyan, Bragg Scattering of Light in Vacuum Structured by Strong Periodic Fields, Phys. Rev. Lett. 107 (2011) 053604 [arXiv:1102.4013].

[57] T. Heinzl, B. Liesfeld, K.-U. Amthor, H. Schwoerer, R. Sauerbrey and A. Wipf, On the observation of vacuum birefringence, Opt. Commun. 267 (2006) 318 [hep-ph/0601076] [INSPIRE].

[58] V. Dinu, T. Heinzl, A. Ilderton, M. Marklund and G. Torgrimsson, Vacuum refractive indices and helicity flip in strong-field QED, Phys. Rev. D 89 (2014) 125003 [arXiv:1312.6419] [INSPIRE].

[59] V. Dinu, T. Heinzl, A. Ilderton, M. Marklund and G. Torgrimsson, Photon polarization in light-by-light scattering: Finite size effects, Phys. Rev. D 90 (2014) 045025 [arXiv: 1405.7291] [INSPIRE].

[60] http://www.hzdr.de/db/Cms?pNid=427\&pOid=35325.

[61] J.T. Mendonça, Axion excitation by intense laser fields, Europhys. Lett. 79 (2007) 21001 [hep-ph/0702091] [INSPIRE].

[62] B. Döbrich and H. Gies, Axion-like-particle search with high-intensity lasers, JHEP 10 (2010) 022 [arXiv:1006.5579] [INSPIRE].

[63] D. Tommasini, A. Ferrando, H. Michinel and M. Seco, Precision tests of QED and non-standard models by searching photon-photon scattering in vacuum with high power lasers, JHEP 11 (2009) 043 [arXiv:0909.4663] [INSPIRE].

[64] S. Villalba-Chávez and A. Di Piazza, Axion-induced birefringence effects in laser driven nonlinear vacuum interaction, JHEP 11 (2013) 136 [arXiv:1307.7935] [INSPIRE].

[65] S. Villalba-Chávez, Laser-driven search of axion-like particles including vacuum polarization effects, Nucl. Phys. B 881 (2014) 391 [arXiv:1308.4033] [INSPIRE]. 
[66] S. Villalba-Chávez and C. Müller, Searching for minicharged particles via birefringence, dichroism and Raman spectroscopy of the vacuum polarized by a high-intensity laser wave, Annals Phys. 339 (2013) 460 [arXiv: 1306.6456] [InSPIRE].

[67] S. Villalba-Chávez and C. Müller, Light dark matter candidates in intense laser pulses I: paraphotons and fermionic minicharged particles, JHEP 06 (2015) 177 [arXiv:1412.4678] [INSPIRE].

[68] V.N. Bă̌er, A.I. Mil'shteĭn and V.M. Strakhovenko, Interaction between a photon and an intense electromagnetic wave, Zh. Eksp. Teo. Fiz. 69 (1975) 1893 [Sov. Phys. JETP 42 (1976) 961].

[69] W. Becker and H. Mitter, Vacuum polarization in laser fields, J. Phys. A 8 (1975) 1638.

[70] S. Meuren, C.H. Keitel and A. Di Piazza, Polarization operator for plane-wave background fields, Phys. Rev. D 88 (2013) 013007 [arXiv: 1304.7672] [INSPIRE].

[71] https://www.gsi.de/en/start/research/forschungsgebiete_und_experimente/ appa_pni_gesundheit/plasma_physicsphelix/phelix.htm.

[72] C. Labaune et al., Fusion reactions initiated by laser-accelerated particle beams in a laser-produced plasma, Nature Commun. 4 (2013) 2506.

[73] M. Ahlers, H. Gies, J. Jaeckel and A. Ringwald, On the Particle Interpretation of the PVLAS Data: Neutral versus Charged Particles, Phys. Rev. D 75 (2007) 035011 [hep-ph/0612098] [INSPIRE].

[74] S. Villalba-Chávez and C. Müller, Photo-production of scalar particles in the field of a circularly polarized laser beam, Phys. Lett. B 718 (2013) 992 [arXiv:1208.3595] [INSPIRE].

[75] J.L. Richard, Group-theoretical analysis of elementary particles in an external electromagnetic field. 3. the relativistic particle in a circularly polarized plane wave and its mass shift, Nuovo Cim. A 8 (1972) 485 [inSPIRE].

[76] S.J. Orfanidis, Electromagnetic Waves and Antennas, Chapter 4, online-book http://www.ece.rutgers.edu/ orfanidi/ewa/.

[77] K. Muroo et al., Measurement of the Cotton-Mouton constants of noble atoms, J. Opt. Soc. Am. B 20 (2003) 2249.

[78] H. An, M. Pospelov and J. Pradler, New stellar constraints on dark photons, Phys. Lett. B 725 (2013) 190 [arXiv:1302.3884] [INSPIRE].

[79] J. Redondo and G. Raffelt, Solar constraints on hidden photons re-visited, JCAP 08 (2013) 034 [arXiv: 1305.2920] [INSPIRE]. 